This document is confidential and is proprietary to the American Chemical Society and its authors. Do not copy or disclose without written permission. If you have received this item in error, notify the sender and delete all copies.

\title{
Effect of Single-Point Mutations on Nitric Oxide Rebinding and the Thermodynamic Stability of Myoglobin
}

\begin{tabular}{|c|c|}
\hline Journal: & The Journal of Physical Chemistry \\
\hline Manuscript ID & jp-2018-11454j.R1 \\
\hline Manuscript Type: & Article \\
\hline $\begin{array}{r}\text { Date Submitted by the } \\
\text { Author: }\end{array}$ & 30-Jan-2019 \\
\hline Complete List of Authors: & $\begin{array}{l}\text { Diamantis, Polydefkis; Swiss Federal Institute of Technology of Lausanne } \\
\text { (EPFL), Institute of Chemical Sciences and Engineering } \\
\text { El Hage, Krystel; Universitat Basel, Department of Chemistry } \\
\text { Meuwly, Markus; Universitat Basel, Chemistry }\end{array}$ \\
\hline
\end{tabular}

\section{SCHOLARONE Manuscripts}




\title{
Effect of Single-Point Mutations on Nitric Oxide
} Rebinding and the Thermodynamic Stability of

\section{Myoglobin}

\author{
Polydefkis Diamantis, ${ }^{\dagger, \ddagger}$ Krystel el Hage $^{\dagger}$ and Markus Meuwly*,†, \\ Department of Chemistry, University of Basel, Klingelbergstrasse 80, 4056 Basel, \\ Switzerland, Current address: Laboratory of Computational Chemistry and Biochemistry, \\ Ecole Polytechnique Fédérale de Lausanne (EPFL), CH-1015 Lausanne, Switzerland, and \\ Department of Chemistry, Brown University, Providence (RI), USA \\ E-mail: m.meuwly@unibas.ch
}

\footnotetext{
*To whom correspondence should be addressed

${ }^{\dagger}$ Department of Chemistry, University of Basel, Klingelbergstrasse 80, 4056 Basel, Switzerland

${ }^{\ddagger}$ Current address: Laboratory of Computational Chemistry and Biochemistry, Ecole Polytechnique Fédérale de Lausanne (EPFL), CH-1015 Lausanne, Switzerland

IDepartment of Chemistry, Brown University, Providence (RI), USA 
January 30, 2019

\begin{abstract}
The effect of single amino-acid mutations on the rebinding dynamics of nitrogen monoxide (NO) to myoglobin is investigated using reactive molecular dynamics simulations. In particular, mutations of residues surrounding the heme-active site (Leu29, His64, Val68) were considered. Consistent with experiments, all mutations studied here have a significant effect on the kinetics of the NO rebinding process which consists of a rapid (several $10 \mathrm{ps}$ ) and a slow (100s of ps) time scale. For all modifications considered the timescales and rebinding fractions agree to within a few percent with results from experiments by adjusting one single, physically meaningful, conformationally averaged quantity: the asymptotic energy separation between the NO-bound $\left({ }^{2} \mathrm{~A}\right)$ and photodissociated $\left({ }^{4} \mathrm{~A}\right)$ states. It is furthermore shown that the thermodynamic stability of WT versus mutant $\mathrm{Mb}$ for the ligand-free- and ligand-bound variants of the protein can be described by the same computational model. Therefore, ligand kinetics and thermodynamics are related in a direct fashion akin to $\Phi$-value analysis which establishes a relationship between protein folding rates and thermal stability of proteins.
\end{abstract}

Keywords: Myoglobin, Mutations, Molecular Dynamics Simulations, Nitrogen Monoxide Rebinding, Thermodynamic Stability 


\section{Introduction}

With its ability to bind and transport diatomics such as oxygen $\left(\mathrm{O}_{2}\right)$, nitrogen monoxide (NO) or carbon monoxide $(\mathrm{CO})$, myoglobin $(\mathrm{Mb})$ plays important physiological functions in living organisms. Its main function consists of binding $\mathrm{O}_{2}$ and storing it in the muscle cells. In addition, myoglobin also reacts with $\mathrm{NO}$ and $\mathrm{CO}$, both of which are involved in processes such as the regulation of blood pressure, neurotransmission and platelet aggregation. This has led to extensive investigations of the interaction of myoglobin with these three diatomics within a physicochemical perspective. ${ }^{1-5}$

Rebinding of NO to wild-type and mutant myoglobins has been extensively investigated, both experimentally ${ }^{6-16}$ and computationally. ${ }^{17-22}$ The studies for WT myoglobin establish that the overall rebinding process consists of a rapid and a slow process. The time constant for the fast process ranges from 12.0 ps to 24.5 ps whereas the slow process extends from 126.4 ps to 279.3 ps. Previous studies on NO rebinding to mutant myoglobins (e.g. V68F, ${ }^{23,24} \mathrm{H} 64 \mathrm{~V}^{14}$ and $\mathrm{H}_{64 \mathrm{Q}^{8}}$ ) suggest that this is also true for mutant Mbs, albeit with different time constants.

In the present work, the rebinding kinetics of NO to WT and mutant $\mathrm{Mb}$ (Figure 1) is studied by means of reactive molecular dynamics (MD) simulations. A particular focus is on investigating the effect of single point amino acid mutations around the ligand binding site (the heme-iron, see Figure 1) on the kinetics of NO rebinding. Here, mutations of Leu29, His64 and Val68 are considered for which experimental reference data is available. This leads to a computational model which can be further assessed in view of other physicochemical properties such as the impact of each mutation on the thermodynamic stability of the ligand-free and ligand-bound-protein. The effect of a mutation on the thermodynamic stability of a protein is most commonly determined by comparing the relative difference in the free energy of unfolding $(\Delta \Delta G)$ for the wild type vs the mutant protein, no ligand included. 


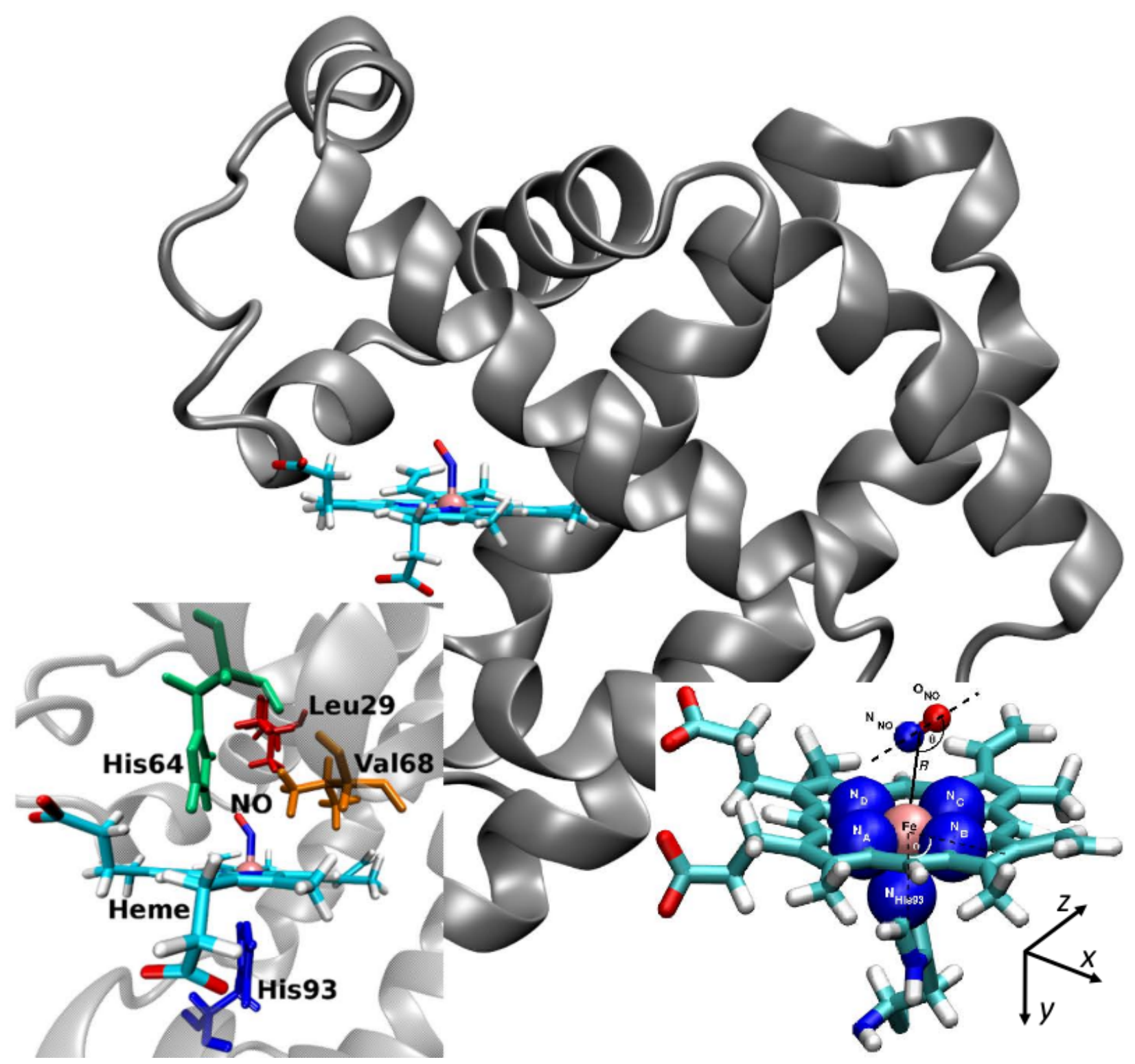

Figure 1: Myoglobin (grey cartoon representation) with the heme group and the bound $\mathrm{NO}$ ligand in the Fe-NO conformation. Heme and NO are shown in licorice. Left-hand inset shows active site environment with His64, Leu29, Val68, and His93 (coordinating from below). Right-hand inset: Internal coordinates $R, \theta$ and $\phi$ to describe the interaction of NO with the heme-iron and the iron out-of-plane motion. The heme unit and the His93 amino acid are shown in licorice, the NO ligand in CPK and the heme-Fe, heme-nitrogen and the nitrogen of the His93 in van der Waals representation.

Previous research ${ }^{25}$ suggests that, for lysozyme, $\Delta \Delta G$ is of the order of $2 \mathrm{kcal} / \mathrm{mol}$. A recent collection of all $\Delta \Delta G$ values shows that they are of similar magnitude for $\mathrm{Mb}^{26}$ 
The connection between kinetics and thermodynamics following structural perturbations has been discussed in the literature in the context of rate-equilibrium-free-energy relationships ${ }^{27}$ following work by Brønsted and Pedersen ${ }^{28}$ and Hammett. ${ }^{29}$ Such linear free energy relationships were then further developed and led, e.g., to $\Phi$-value analysis which relates the folding kinetics and thermodynamic stability of the wild-type protein with that of point mutants, e.g. from protein engineering. ${ }^{30,31}$ Here, the kinetics for ligand rebinding and the stability changes between ligand-bound- and ligand-free WT and single-point mutants for $\mathrm{Mb}$ is analyzed based on a molecularly refined picture for ligand rebinding dynamics.

In the following, first the computational methods are described. Then, the NO-rebinding dynamics for WT and a number of experimentally characterized Mb-mutants is discussed, followed by an analysis of the thermodynamic stability of the ligand-free and ligand-bound WT and mutant Mbs. Finally, the results from kinetics and thermodynamics are discussed, and then conclusions are drawn.

\section{Computational Methods}

All MD simulations were carried out using the CHARMM program ${ }^{32,33}$ with the all atom force field for proteins CHARMM22, ${ }^{34}$ including the CMAP correction, ${ }^{35}$ iron in its +2 oxidation state (Fe(II)), and periodic boundary conditions. The wild-type (WT) and 12 mutant myoglobins were studied: L29F, L29V, L29W, H64A, H64Q, H64V, H64L, H64F, V68F, V68L, V68I, and V68W.

In each case, the system consisted of the NO-bound protein in the Fe-NO conformation, solvated in a cubic box of TIP3P $\mathrm{P}^{36}$ water, with dimensions $63.6 \times 63.4 \times 63.5 \AA^{3}$. The systems were heated to $300 \mathrm{~K}$, equilibrated at $300 \mathrm{~K}$ and 1 atm for $250 \mathrm{ps}$, and further propagated 
for another $100 \mathrm{ps}$, using a timestep of 1 fs and a cutoff of $14 \AA$ for the non-bonded interactions. For electrostatic interactions, Particle mesh Ewald (PME) was used with grid-size spacing of $1 \AA$ and a relative tolerance of $10^{-6}$. SHAKE was used for constraining the bonds involving hydrogen atoms. ${ }^{37,38}$ In order to generate a diverse set of initial conditions for the subsequent reactive MD simulations, 5 independent initialization runs were performed.

\subsection{NO Rebinding Simulations}

Reactive MD simulations were performed using accurate and validated 3-dimensional potential energy surfaces $V(R, \theta, \phi)$ for the bound ${ }^{2} \mathrm{~A}$ and unbound ${ }^{4} \mathrm{~A}$ states. ${ }^{21,22}$ This PES is a reproducing kernel Hilbert space (RKHS) representation ${ }^{39,40}$ based on more than 1000 energies calculated at the B3LYP/6-31G(d,p) level of theory. The relevant coordinates are the Fe-CoM(NO) coordinate $R$, the angle $\theta$ between the Fe-CoM(NO) and the NO vectors, and the Fe-out-of plane position angle $\phi$, see inset Figure 1. Both states relevant to the present work (the bound ${ }^{2} A$ and unbound ${ }^{4} A$ manifold) are used together with reactive molecular dynamics simulations in order to follow the rebinding dynamics after NO-photodissociation from the heme-iron.

The asymptotic separation: A priori the two force fields for the ${ }^{2} A$ and ${ }^{4} A$ states are independent of each other because they do not share a common zero of energy. However, their asymptotic energy separation $\Delta$ (see Figure 2) is known to be between 5 and $10 \mathrm{kcal} / \mathrm{mol} .^{22,41}$ The asymptotic energy separation is the energy difference between the ${ }^{4} A$ and ${ }^{2} A$ states for infinite separation of the ligand and asymptotically, the ${ }^{4} A$ state is the lower of the two (see Figure 2.) This has to be taken into account when using the two force fields in a reactive MD simulation because for this the relative energy separation is relevant. For WT Mb, an appropriate value to qualitatively describe NO-rebinding with the mixed RKHS/CHARMM22 force field was found to be $\Delta=-10.25 \mathrm{kcal} / \mathrm{mol} .{ }^{21,22}$ However, this value does not provide 
the correct rebinding kinetics and depending on the active site environment, this value may change due to local interactions. Furthermore, such a $\Delta$ is a conformationally averaged quantity.

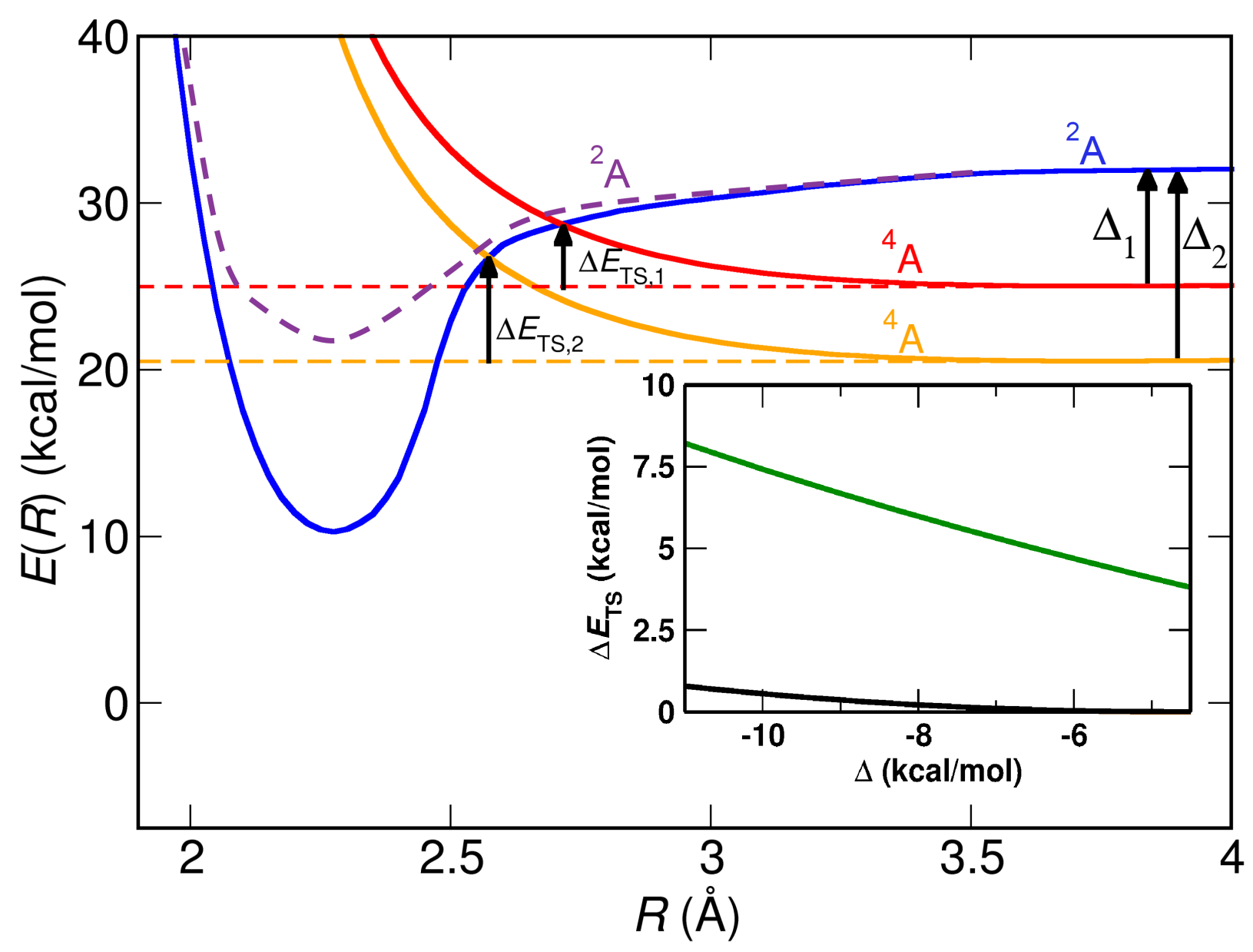

Figure 2: Schematic to show the relationship between the ${ }^{2} A$ (Mb-NO bound state, solid blue and $\mathrm{Mb}-\mathrm{ON}$ bound state, dashed blue) curves and two ${ }^{4} A$ (unbound state, red and orange) curves for different values of $\Delta_{1}$ and $\Delta_{2}$. These two asymptotic shifts lead to different inner barriers $\Delta E_{\mathrm{TS}, 1}$ and $\Delta E_{\mathrm{TS}, 2}$ which affect the rebinding dynamics and to stabilization of the Mb-ON state (for $\Delta_{1}$ ) or not (for $\Delta_{2}$ ). The inset shows the relationship between the value of $\Delta$ and the height of the inner barrier $\Delta E_{\mathrm{TS}}$ for the Fe-NO (black) and the Fe-ON configuration (green), respectively.

NO-Rebinding Protocol: Following the initial equilibration for each system five independent runs were continued in the $N V T$ ensemble $(300 \mathrm{~K})$ for $2 \mathrm{~ns}$ in the NO-bound state, using the 
${ }^{2} A$ RKHS potential. Every $2.5 \mathrm{ps}$, a frame is extracted for which a constant energy $(N V E)$ MD simulation is successively run in (i) the bound state for $2.5 \mathrm{ps,} \mathrm{(ii)} \mathrm{the} \mathrm{Fe-NO} \mathrm{bond} \mathrm{is}$ broken and the dynamics is continued on the unbound state for $0.5 \mathrm{ps}$ (which is the typical time until the unliganded species appears) and (iii) followed by dynamics on the mixed, reactive PES for a maximal simulation time of 200 ps. Excitation from the bound to the unbound state (step (ii)) is done by instantaneously switching the force fields from ${ }^{2} A$ to ${ }^{4} A$. This adds approximately $50 \mathrm{kcal} / \mathrm{mol}$ to the system which is within the range of excitation energies $\left(49 \mathrm{kcal} / \mathrm{mol}\right.$ to $81 \mathrm{kcal} / \mathrm{mol}$ ) used in experiments. ${ }^{42}$ If the ligand rebinds in the Fe-NO conformation earlier than 200 ps after photodissociation, the simulation is stopped. However, it is also possible that the ligand transiently rebinds in the Fe-ON geometry from where further dynamics may lead to Fe-NO. For WT Mb a final Fe-ON geometry was found for $2 \%$ of the trajectories with $\Delta=-7.25 \mathrm{kcal} / \mathrm{mol}$ and no stable Fe-ON state was obtained with $\Delta=-10.25 \mathrm{kcal} / \mathrm{mol}$.

Data Analysis: For trajectories in which NO rebinds within the total simulation time allowed (200 ps), a rebinding time is determined. The ensemble of rebinding times provides the basis to follow the rebinding kinetics. For this, the cumulative distribution $p(\tau)$ of rebinding times is determined, from which the unbound fraction $N(t)$ can be obtained. Then, rebinding times are determined based on the kinetic constants extracted from fitting to single- or multipleexponential decays

$$
N(t)=\sum_{i} a_{i} e^{-\left(k_{i} t\right)}
$$

where $k_{i}$ are the kinetic coefficients and $a_{i}$ is the associated amplitude for process $i$. The respective decay times are then $\tau_{i}=1 / k_{i}$. Sums of exponentials were used to allow direct comparison with the literature. ${ }^{7,12,16,22,23}$ However, other parametrizations have also been used in the past, in particular stretched exponentials $N(t)=a e^{-(k t)^{\beta}}$ or a power-law. ${ }^{6}$ 


\subsection{Free Energy Simulations}

Computational mutations to estimate protein stability is based on the notion that the binding free energy corresponding to a given side chain, considered as a "pseudo-ligand" of the wild type protein, reflects the importance of this side chain to the thermodynamic stability of the protein. ${ }^{43}$ The protein stability difference $\Delta \Delta G_{\text {stab }}$ between WT and a particular mutant $\mathrm{Mb}$ was calculated according to $\Delta \Delta G_{\text {stab }}=\Delta G_{2}-\Delta G_{1}=\Delta G_{4}-\Delta G_{3}$ (see Figure 3 ) since it is computationally easier to determine $\Delta G_{1}$ and $\Delta G_{2}$ than $\Delta G_{3}$ and $\Delta G_{4} \cdot{ }^{44}$ Here, $\Delta G_{1}$ and $\Delta G_{2}$ are the free energies for changing the side chain of a WT residue (WT a.a.) into its mutant in aqueous phase as an isolated residue $\left(-\mathrm{CH}_{3}\right.$ terminated at $\left.\mathrm{C} \beta\right)$ and in the protein, respectively. Therefore, destabilizing mutations have a positive $\Delta \Delta G$.

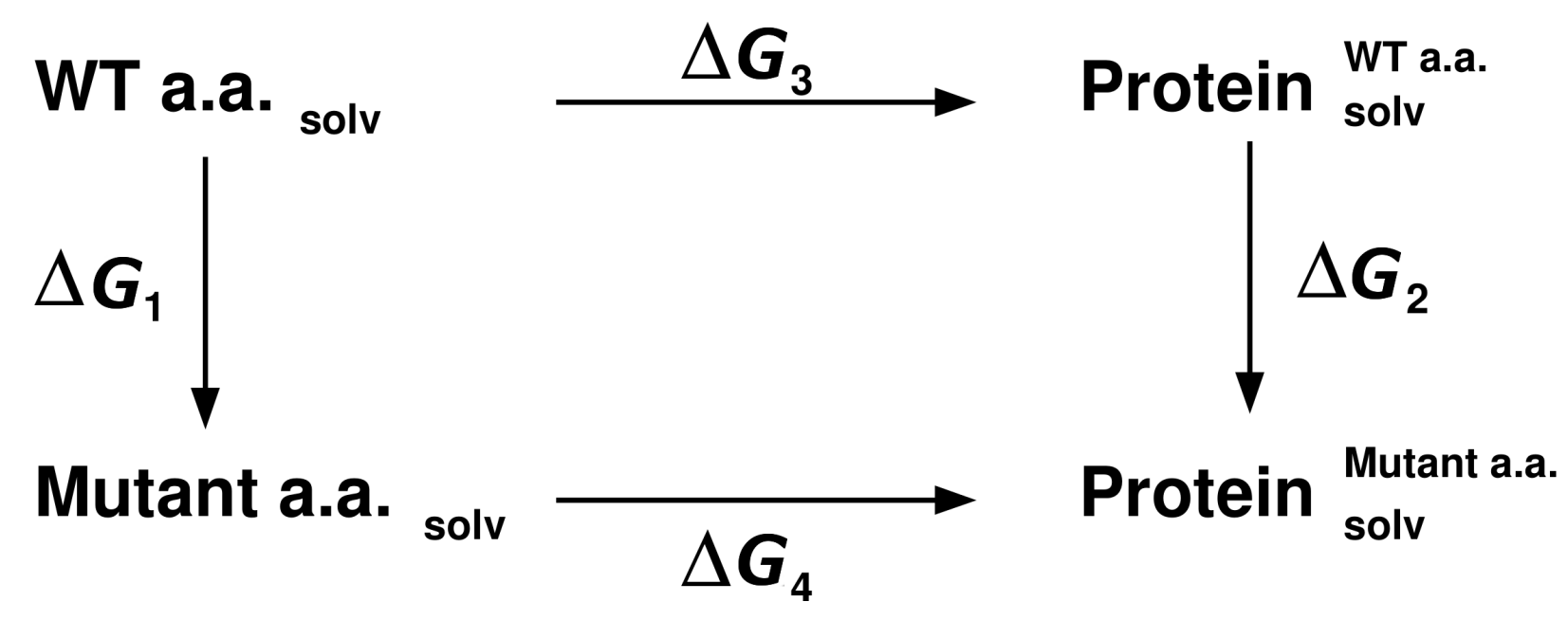

Figure 3: Thermodynamic cycle used to calculate the stability free energy differences $\Delta \Delta G_{\text {stab }}=\Delta G_{2}-\Delta G_{1}=\Delta G_{4}-\Delta G_{3}$ between WT protein and the mutants. $\Delta G_{1}$ and $\Delta G_{2}$ are the free energies of mutating the side chain of the residue in question in aqueous phase as an isolated residue and in the protein, respectively. And $\Delta G_{3}$ and $\Delta G_{4}$ are the free energies of going from a sole residue to being part of the protein for the WT residue and for the mutant, respectively.

For the free energy simulations, hybrid residues with dual topology ${ }^{44,45}$ were constructed for mutations L29V, H64F, H64V and V68F, both in their unbound- and NO-bound forms. Initial coordinates were taken from the previously equilibrated structures. 
All contributions to the free energies in the thermodynamic cycle were computed using thermodynamic integration (TI ${ }^{46}$ which applies a scaling parameter $\lambda$, and switches between the initial $(\lambda=0$, state $\mathrm{A})$ and the final $(\lambda=1$, state $\mathrm{B})$ state by gradually damping all nonbonded interactions. The free energy difference is then

$$
\Delta G_{\mathrm{A} \rightarrow \mathrm{B}}=\int_{0}^{1} \mathrm{~d} \lambda\left\langle\frac{\partial \mathcal{H}}{\partial \lambda}\right\rangle_{\lambda} \approx \sum_{i}\left(\lambda_{i+1}-\lambda_{i}\right)\left\langle\frac{\partial \mathcal{H}}{\partial \lambda}\right\rangle_{\lambda_{m}}
$$

where $\mathrm{A} \rightarrow \mathrm{B}$ refers to the transformation between the WT (state A) and the mutant (state B). The canonical average $\langle\cdot\rangle_{\lambda}$ is performed over the phase space generated by $\mathcal{H}(\lambda)$, and $\lambda_{m}=\left(\lambda_{i}+\lambda_{i+1}\right) / 2$. The calculation is performed using the PERT module in CHARMM along with soft-core potentials for the Lennard-Jones contribution. ${ }^{46,47}$

Free energy simulations at each $\lambda$-value were carried out in the NPT ensemble, using the Hoover heat-bath method ${ }^{48}$ with pressure coupling at $T=298 \mathrm{~K}, p=1 \mathrm{~atm}$, and the masses of the temperature and pressure piston to $20 \%$ and $2 \%$ of the system's mass, respectively. A friction coefficient of $50 \mathrm{ps}^{-1}$ was used. The interval $0<\lambda<1$ was divided into 34 steps with windows at the two ends of the $\lambda$-interval more finely spaced. ${ }^{49}$ For each of these steps the system was re-equilibrated for 20 ps followed by 40 ps of dynamics during which information was accumulated. $\lambda$ was changed from initial to final value using the slow-growth protocol, ${ }^{50}$ which allowed the system to re-equilibrate between steps. The overall results from the forward (WT $\rightarrow$ Mutant) and backward (Mutant $\rightarrow$ WT) run are lower and upper bounds to the free energy difference, respectively. The results reported are averages of 5 runs. 


\section{Results and Discussion}

\subsection{Rebinding Dynamics}

For an initial assessment of the rebinding kinetics, reactive MD simulations were carried out with a shift of $\Delta=-10.25 \mathrm{kcal} / \mathrm{mol}$ which had been determined in previous work. ${ }^{22}$ The unbound fraction $N(t)$ of WT and mutant Mbs after photodissociation from 750 independent trajectories, each propagated for 200 ps or less if the ligand rebound earlier, are shown in Figure 4. The rebound fraction after 200 ps is $60.0 \%$ (see Table 1) for WT and differs substantially for the various mutants.

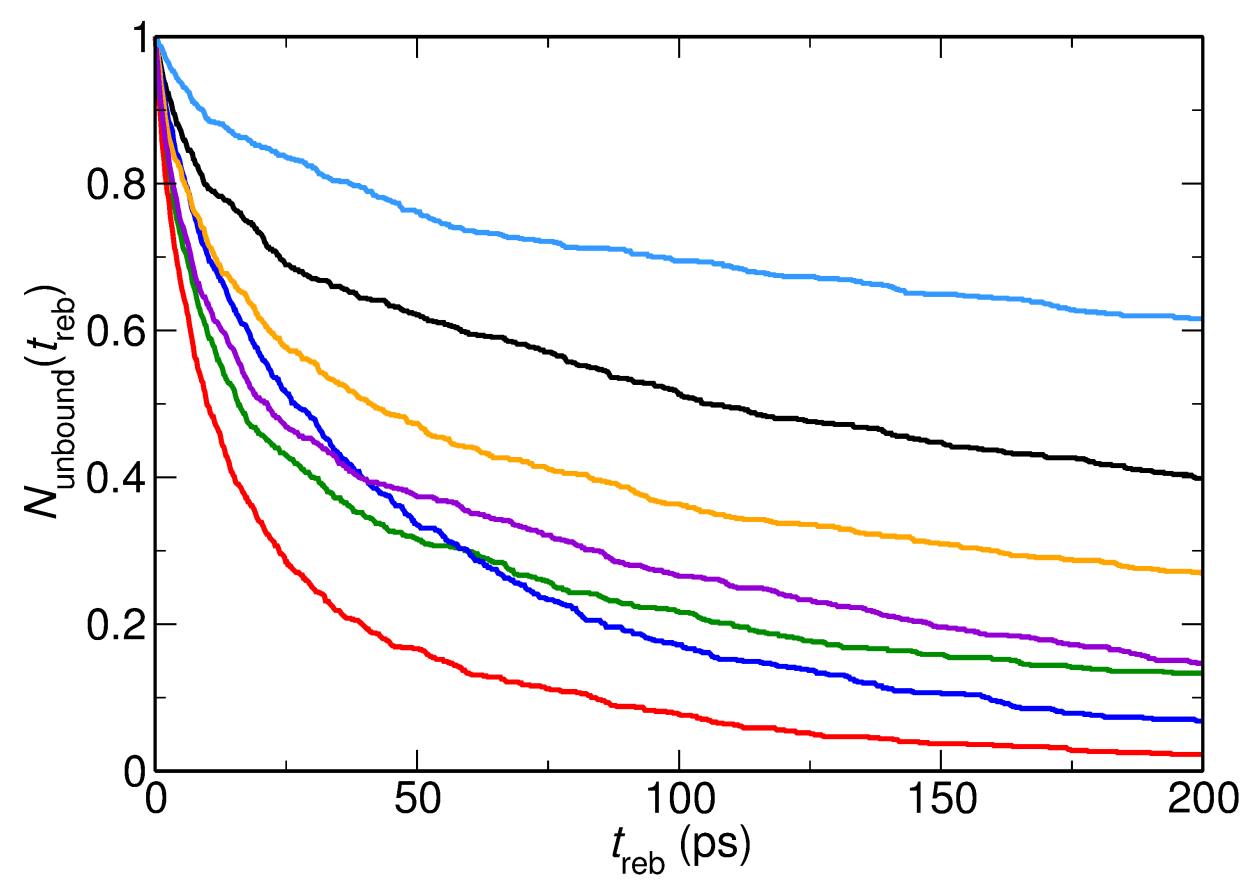

Figure 4: NO rebinding curves from 750 independent simulations for WT and mutant myoglobins with $\Delta=-10.25 \mathrm{kcal} / \mathrm{mol}$; WT (black), H64Q (red), H64V (green), V68F (blue) V68I (orange), L29F (violet) and L29V (cyan).

Table 1 reports the decay times for wild-type and several mutant Mbs, the corresponding amplitudes, as well as the rebinding fractions after 200 ps compared with experiments. For WT myoglobin, $\tau_{1}$ lies at the lower bound of values reported in the literature (for detailed 
Table 1: NO-rebinding times $\tau_{i}$, amplitudes $a_{i}$ and the fraction of rebound $N$ at $200 \mathrm{ps}$ for WT and mutant Mbs from simulations with $\Delta=-10.25 \mathrm{kcal} / \mathrm{mol}$. Results from the present work and from the literature are compared. References to experiments for WT, ${ }^{6,8,10,14,23,24,51,52} \mathrm{H64Q},{ }^{8} \mathrm{H64V},{ }^{14} \mathrm{~V} 68 \mathrm{~F},{ }^{23,24,53} \mathrm{~V} 68 \mathrm{I},{ }^{24,53}$ $\mathrm{L29F},{ }^{7}$ and L29V. ${ }^{7}$ For amplitudes from analysis of experiments which do not not add up to 1 , the fitting function included a constant offset $a_{m}$, i.e. $N(t)=$ $\sum_{i=1}^{m-1} a_{i} \exp ^{-t / \tau_{i}}+a_{m}$.

\begin{tabular}{|l|r|r|r|r|r|}
\hline Mb & $a_{1}(\mathrm{ps})$ & $\tau_{1}(\mathrm{ps})$ & $a_{2}(\mathrm{ps})$ & $\tau_{2}(\mathrm{ps})$ & $\% N(t)(200 \mathrm{ps})$ \\
\hline WT & 0.42 & 11.4 & 0.58 & 326.5 & $60.0 \pm 1.9$ \\
Experiment & 0.37 to 0.54 & 12.0 to 24.5 & 0.46 to 0.63 & 126.4 to 279.3 & 77.0 to 81.2 \\
\hline H64Q & 0.63 & 8.1 & 0.37 & 63.5 & $97.7 \pm 0.6$ \\
Experiment & 0.48 & 8.9 & 0.43 & 122.0 & 82.7 \\
\hline H64V & 0.55 & 9.2 & 0.45 & 144.2 & $86.6 \pm 1.4$ \\
Experiment & 0.51 & 10.0 & 0.43 & 92.0 & 89.1 \\
\hline V68F & 0.42 & 14.0 & 0.58 & 85.0 & $93.2 \pm 1.1$ \\
Experiment & 0.78 & 8.0 to 10.1 & 0.21 & 35.0 to 60.2 & 98.3 \\
\hline V68I & 0.45 & 13.0 & 0.55 & 259.5 & $73.2 \pm 1.9$ \\
Experiment & 0.36 & 91.0 & 0.33 & 955.0 & 98.3 \\
\hline L29F & 0.48 & 8.4 & 0.52 & 155.5 & $85.4 \pm 1.5$ \\
Experiment & 0.95 & 6.0 to 6.7 & 0.03 & 200.0 & 96.9 \\
\hline L29V & 0.21 & 20.9 & 0.79 & 777.8 & $38.5 \pm 2.5$ \\
Experiment & & & 0.80 & 400.0 & 31.9 \\
\hline
\end{tabular}

list of references, see Table 1), while $\tau_{2}=327 \mathrm{ps}$ is larger than the largest value reported in previous experimental studies (280 ps). ${ }^{6}$ The rebound fraction after $200 \mathrm{ps}(60 \%)$ is considerably lower compared to the range of values found in the literature $(77.0 \%$ to $81.2 \%)$. This disagreement and how to resolve it will be further discussed when considering how these observables depend on the value of the asymptotic shift $\Delta$ between the ${ }^{2} \mathrm{~A}$ and ${ }^{4} \mathrm{~A}$ states.

The kinetics of NO rebinding to Mb has also been studied experimentally for the mutants H64Q $,{ }^{8} \mathrm{H} 64 \mathrm{~V},{ }^{14} \mathrm{~V} 68 \mathrm{~F},{ }^{23,24,53} \mathrm{~V} 68 \mathrm{I},{ }^{24,53} \mathrm{~V} 68 \mathrm{~L},{ }^{53} \mathrm{~L} 29 \mathrm{~F},{ }^{7}$ and L29V ${ }^{7}$ The results in Table 1 from rebinding simulations with $\Delta=-10.25 \mathrm{kcal} / \mathrm{mol}$ indicate that for the $\mathrm{H} 64 \mathrm{~V} \mathrm{mu}-$ tant close agreement with experiment is found for rebinding times $\left(\tau_{1}, \tau_{2}\right)$, their amplitudes $\left(a_{1}, a_{2}\right)$ and the rebound fraction $N(t)$ after 200 ps. Furthermore, most of the short rebinding times $\tau_{1}$ (except for V68I) are consistent with experiment whereas the amplitude $a_{1}$ of 


\subsection{Influence of the Asymptotic Shift}

As previously discussed, for most myoglobins with decay times/rebinding fractions available in the literature, the reactive MD simulations were not able to quantitatively describe rebinding times, amplitudes and fractions within 200 ps. The only free parameter in the present simulations is the asymptotic separation $\Delta$ between the ${ }^{2} A$ and the ${ }^{4} A$ state. To illustrate the effect of $\Delta$, the potential energy curve for a typical situation (NO-heme-histidine, $\theta=150^{\circ}$ ) is sketched in the main part of Figure 2. Depending on the value of $\Delta$ the crossing geometry and barrier height between the two manifolds varies. The inset of Figure 2 shows the effect of $\Delta$ on the rebinding barrier height $\Delta E_{\mathrm{TS}}$ for the Fe-NO and Fe-ON configurations $\left(\theta=150^{\circ}\right.$ and $\theta=30^{\circ}$, respectively). For the Fe-NO configuration, the barrier is less than $\approx 2 \mathrm{kcal} / \mathrm{mol}$ for a wide range of $\Delta$. This is consistent with the notion that NO rebinding to heme Fe via the $\mathrm{N}$ atom is thermodynamically an almost barrier-less process. ${ }^{54}$ On the contrary, transition barriers for the Fe-ON configuration depend more strongly on $\Delta$. 
Except for H64V (see Table 1) the simulations performed with $\Delta=-10.25 \mathrm{kcal} / \mathrm{mol}$ fail to reproduce the experimentally observed kinetics for NO rebinding to all Mbs. This implies that - as a consequence of the modified active site environment for each mutant - the value of $\Delta$ required to match the experimentally observed rebinding times, amplitudes and fraction slightly differs from one Mb variant to another.

As an example, for wild-type Mb, the experimentally observed rebound fraction $(77.0 \%$ to $81.2 \%$ ) differs from the computed value $(60 \%)$ with $\Delta=-10.25 \mathrm{kcal} / \mathrm{mol}$. Hence, the shift was modified to $\Delta=-7.25 \mathrm{kcal} / \mathrm{mol}$. From 1750 rebinding trajectories the rebound fraction after 200 ps is $78.1 \%$, with decay times of 12.9 ps and 202.9 ps and amplitudes of 0.45 and 0.55 , respectively. These results are i) a clear improvement over the simulations with $\Delta=-10.25 \mathrm{kcal} / \mathrm{mol}$, and ii) all within the ranges reported from experiments on WT myoglobin (see Table 1) and with recent X-ray absorption experiments. ${ }^{16}$ The raw simulation data (open symbols), together with the double exponential fit (red), are shown in Figure 5 together with experimentally determined NO-rebinding curves to Mb in water 6,8,10,14,23,24,51,52 and the agreement is found to be excellent.

It is also possible to directly compare with experiments at $T=283 \mathrm{~K}$, carried out in $\mathrm{D}_{2} \mathrm{O} .{ }^{12}$ The viscosities of $\mathrm{H}_{2} \mathrm{O}$ and $\mathrm{D}_{2} \mathrm{O}$ differ by $\sim 20 \%^{55}$ and lowering the temperature leads to an increase in viscosity in the temperature range between 290 and $200 \mathrm{~K}$, respectively. ${ }^{52}$ Earlier experiments had pointed towards a solvent-viscosity dependence for NO rebinding to Mb on the sub-nanosecond time scale in glycerol/water mixtures ${ }^{9}$ and also in microperoxidase. ${ }^{12,56}$ Also, the NMR spectrum of ligand-bound $\mathrm{Mb}$ was found to depend on the $\mathrm{H}_{2} \mathrm{O} / \mathrm{D}_{2} \mathrm{O}$ ratio used in the experiments ${ }^{57}$ as was the electron transfer rate in Mb. ${ }^{58}$ Finally, the infrared spectroscopy of $\mathrm{MbCO}$ also showed a dependence on the $\mathrm{H}_{2} \mathrm{O} / \mathrm{D}_{2} \mathrm{O}$ ratio. ${ }^{59}$ Therefore, additional reactive MD simulations were performed for NO rebinding to wild-type myoglobin at $283 \mathrm{~K}$ which is the temperature used in the experiments. ${ }^{12}$ In total, 750 rebinding simulations 


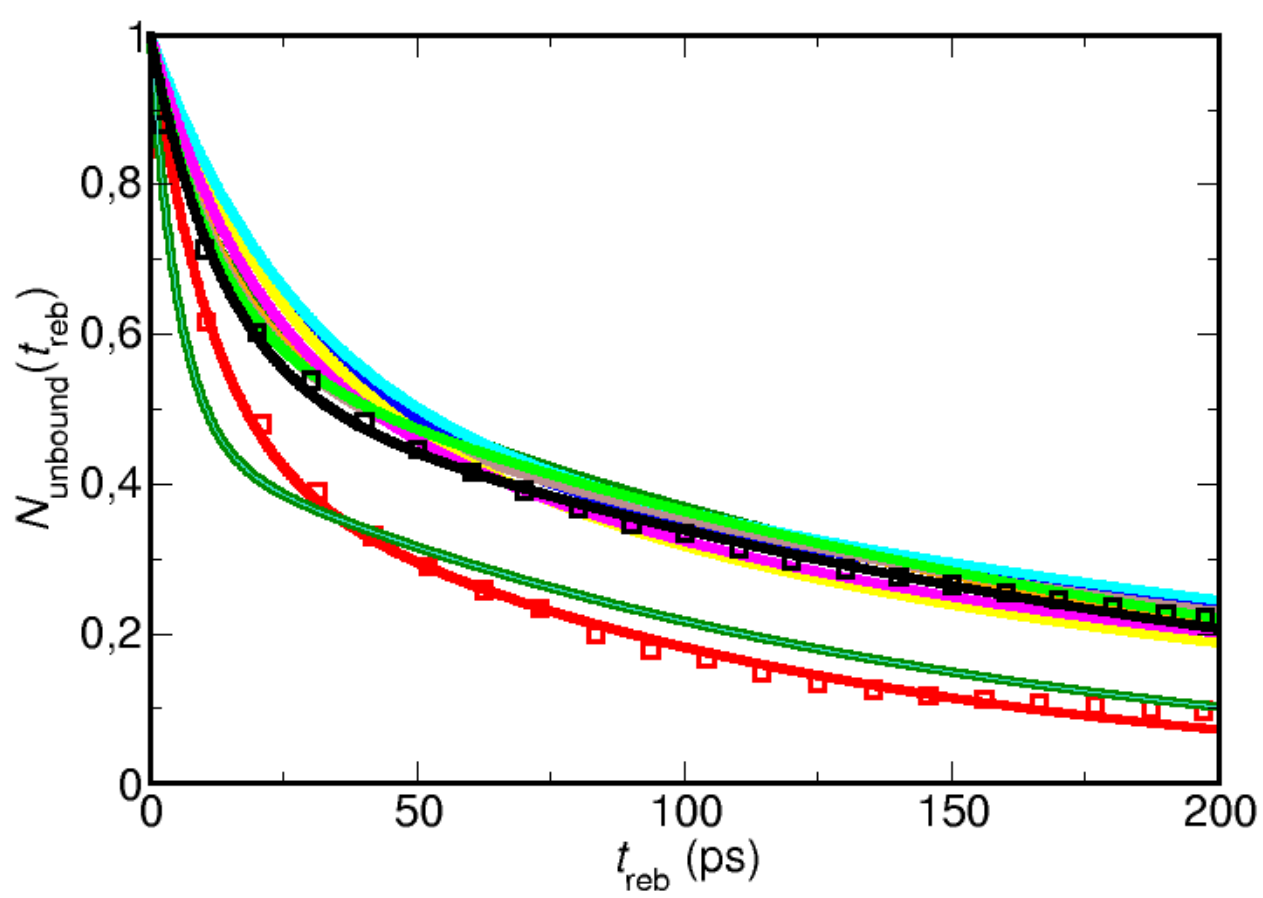

Figure 5: The NO-rebinding curves for WT Mb for $\Delta=-7.25 \mathrm{kcal} / \mathrm{mol}$ compared with experimental data. The open symbols (black for rebinding in $\mathrm{H}_{2} \mathrm{O}$; red in $\mathrm{D}_{2} \mathrm{O}$ ) are the raw data and the solid black and red curves are the fits to a bi-exponential decay, respectively. Experimental kinetics are all colored curves using the parameters from the literature. The dark green trace is for rebinding in $\mathrm{D}_{2} \mathrm{O}^{12}$ and all other traces are for rebinding in $\mathrm{H}_{2} \mathrm{O} .6,8,10,14,23,24,51,52$

were run and analyzed. The rebinding fraction was $90.3 \%$, and fitting a double exponential function yields decay times of 11.2 ps and 107.8 ps, respectively, with amplitudes of 0.54 and 0.46 , consistent with the experiments $(89.8 \%, 5.3$ ps and $133.0 \mathrm{ps}$, amplitudes 0.54 and 0.46). ${ }^{12}$ Simulations at $300 \mathrm{~K}$ yield a rebinding fraction of $97.2 \%$ at $200 \mathrm{ps}$, with decay times of $9.5 \mathrm{ps}$ and $68.6 \mathrm{ps}$, respectively, with amplitudes $a_{1}=0.64$ and $a_{2}=0.36$.

For the V68F mutant (see Table 1), the kinetic parameters (rebinding fraction, decay times, amplitudes) determined with $\Delta=-10.25 \mathrm{kcal} / \mathrm{mol}$, were not fully consistent with the experiments. Hence, rebinding simulations were carried out for $\Delta=-7.25 \mathrm{kcal} / \mathrm{mol}$. The 
rebound fraction after 200 ps is $98.4 \%$, in very good agreement with the one found from an experimental fit $(98.3 \%) .{ }^{24}$ Decay times $\tau_{1}$ and $\tau_{2}$ are 8.0 ps and $63.5 \mathrm{ps}$, respectively, also in very good agreement with the literature. ${ }^{23,24}$ whereas the amplitudes determined from the rebinding simulations $(0.55 / 0.45)$ differ somewhat from those obtained from the experimental fit $(0.78 / 0.21)$. Overall, changing $\Delta$ from $-10.25 \mathrm{kcal} / \mathrm{mol}$ to $-7.25 \mathrm{kcal} / \mathrm{mol}$ considerably improved the agreement between simulations and experiment for the WT and the V68F mutant.

Finally, simulations with modified shifts were also performed for the L29F mutant. Two different values of $\Delta$ were considered, $-8.25 \mathrm{kcal} / \mathrm{mol}$ and $-6.25 \mathrm{kcal} / \mathrm{mol}$, respectively. For $\Delta=-8.25 \mathrm{kcal} / \mathrm{mol}$, the rebound fraction after $200 \mathrm{ps}$ was $86.4 \%$, with decay times of 6.4 ps and $145.5 \mathrm{ps}$, and respective amplitudes of 0.56 and 0.44 . For $\Delta=-6.25 \mathrm{kcal} / \mathrm{mol}, N(t)$ after 200 ps amounted to $90.0 \%$, with $\tau_{1}$ and $\tau_{2}$ are 6.6 ps and $132.1 \mathrm{ps}, a_{1}=0.60$ and $a_{2}=0.40$. As $\Delta$ is decreased, the rebound fraction increases, while $\tau_{2}$ decreases and the amplitudes change slightly. Overall, the decrease of the asymptotic shift brings the reactive MD simulations closer to the experimental observations. ${ }^{7,20}$

In summary, the best agreement between computed rebinding fractions and rebinding times for the four systems WT, L29F, H64V, and V68F from explicit rebinding simulations was obtained with asymptotic shifts $\Delta$ ranging from $-10.25 \mathrm{kcal} / \mathrm{mol}$ to $-6.25 \mathrm{kcal} / \mathrm{mol}$. As already mentioned, the value of $\Delta$ is a conformationally averaged quantity. In reality, $\Delta$ depends in a complicated fashion on the instantaneous conformation of the heme-unit and the orientation of the unbound ligand relative to the heme and it would be difficult to cast this dependence in parametrized form. The different "best values" for $\Delta$ for the different mutants highlight the fact that depending on the active site mutation the energetics and thereby the rebinding kinetics are modified, see Figure 2. 


\subsection{Differential Stability of NO-Bound and Unbound Mb}

Because the ligand (NO) always interacts in the same fashion with the heme group to which it binds (because $V(R, \theta, \phi)$ does not change depending on the protein variant), differences in rebinding kinetics for $\mathrm{WT}, \mathrm{L} 29 \mathrm{~F}, \mathrm{H} 64 \mathrm{~V}$, and V68F - and hence chemical selectivity - is exercised through other factors. One possibility is the differential thermodynamic stabilization between the ligand-free and ligand-bound forms of the WT and the mutants. In other words, the relative thermodynamic stability between $\mathrm{WT}$ and mutant $\mathrm{Mb}$ for the ligand-free and ligand-bound state differ due to modifications in the local interactions between the ligand and the environment.

The available experimental data on the stability of holo- and apo-Mb have been recently assessed and analyzed to compare with predictions from protein stability calculators. ${ }^{26}$ In the following, this data (SI Table S1 from Ref. ${ }^{26}$ ) and the present free energy simulations are compared. The analysis ${ }^{26}$ assumes that differential stability changes between WT and 
mutant $\mathrm{Mb}$ are similar for holo- and apo-Mb, respectively. This is consistent with the finding that the bimolecular rate of hemin binding is approximately the same for all apohemoglobins and apomyoglobins ${ }^{60}$ and that hemin loss rates vary only in a narrow range. ${ }^{61}$ For ligandbound MbNO the differential stabilization energies are not well known due to experimental difficulties. ${ }^{26,62-64}$ In particular, for $\mathrm{Mb}(\mathrm{II}) \mathrm{NO}$ (with iron as $\mathrm{Fe}(\mathrm{II})$ ) the unfolding would have to be anaerobically which generates metMb and $\mathrm{NO}_{3}^{-}$whereas with $\mathrm{Fe}(\mathrm{III})$ with air present the NO dissociates which forms nitrites and NO radicals through oxidation. Changes in thermodynamic stability for the mutations L29F, L29V, H64F, H64V and V68F (see Figure 1) in their ligand-free and NO-bound forms were determined from free energy simulations (see Methods) and the results are summarized in Table 2.

Table 2: Protein stability difference (in $\mathrm{kcal} / \mathrm{mol}$ ) for L29F, L29V, H64F, H64V and V68F mutations for ligand-free and ligand-bound $\mathrm{Mb}$. The estimated experimental error is $\approx 0.5 \mathrm{kcal} / \mathrm{mol}^{26} \delta \Delta \Delta G_{\mathrm{stab}}=\Delta \Delta G_{\text {stab }}^{\text {ligand-free }}-\Delta \Delta G_{\text {stab }}^{\text {ligand-bound }}$ is the difference in stability when mutating the residues in question in the ligandfree and in the ligand-bound form, respectively. The reference value for the asymptotic separation is $\Delta_{\mathrm{WT}}=-7.25 \mathrm{kcal} / \mathrm{mol}$ which is the value to obtain the experimentally observed kinetics for WT Mb. From this, the expected (or predicted) $\Delta_{\text {Mut }}$ for a mutant - under the assumption that kinetics and thermodynamic stability are related - is $\Delta_{\text {mut }}=\delta \Delta \Delta G_{\mathrm{stab}}+\Delta_{\mathrm{WT}} \cdot{ }^{*}$ indicates value for $\delta \Delta$ estimated to yield best agreement with NO-rebinding kinetics from simulations of the relative thermodynamic stability.

\begin{tabular}{l|ccccc}
\hline$\Delta \Delta G_{\text {stab }}$ & L29F & L29V & H64F & H64V & V68F \\
\hline Ligand-free & $0.34 \pm 0.4$ & $1.53 \pm 0.31$ & $-0.54 \pm 0.3$ & $-0.56 \pm 0.3$ & $-1.05 \pm 0.3$ \\
Ligand-free (Exp.) & $0.13^{61}$ & $1.72^{65}$ & $-1.51^{61} /-0.81^{66}$ & $-0.36^{66}$ & $-0.38^{66} /-0.82^{61}$ \\
iStable 67 & 1.36 & 1.66 & -0.90 & -0.97 & 0.84 \\
\hline NO-bound & $-0.79 \pm 0.4$ & $1.65 \pm 0.5$ & $5.25 \pm 0.37$ & $2.30 \pm 0.4$ & $-3.40 \pm 0.4$ \\
\hline \hline$\delta \Delta \Delta G_{\text {stab }}$ & 1.1 & -0.1 & -5.8 & -2.9 & 2.4 \\
$\delta \Delta$ & 1 to $2^{*}$ & N.D. & N.D. & $-3.0^{*}$ & $2^{*}$ \\
\hline
\end{tabular}

For L29V the computed destabilization $(1.53 \mathrm{kcal} / \mathrm{mol})$ for ligand-free Mb compares favourably with the experimentally determined value of $1.72 \mathrm{kcal} / \mathrm{mol}$ for holo-Mb. ${ }^{65}$ This serves as a benchmark for the free energy simulations. The ligand-bound form is also destabilized by 
a similar amount (1.65 kcal/mol, see Table 2). Thus, for L29V the differential change in thermodynamic stability $\delta \Delta \Delta G_{\text {stab }} \approx 0$.

For L29F - replacing Leu with the larger Phe (hydrophobic) residue - a negligible stability change is found for the ligand-free form $(0.34 \mathrm{kcal} / \mathrm{mol}$ compared with $0.13 \mathrm{kcal} / \mathrm{mol}$ for the apo protein ${ }^{26,61}$ ) whereas the NO-bound protein is stabilized by $-0.8 \mathrm{kcal} / \mathrm{mol}$. This mutation affects the volume, the geometry of the binding site and the internal hydrophobic side chain packing, see Figure 6. A structural explanation is provided by separate 10 ns of MD simulations for the ligand-free and ligand-bound forms for the WT and L29F mutation (Figure 6). Superposition of the WT ligand-free and ligand-bound structure at 0, 5 and 10 ns of MD shows only minor structural changes in the active site (Figure 6, upper row). However, replacing the highly conserved Leu29 by a bulky phenylalanine side chain decreases the volume of the binding site. This leads to frequent collisions between the bound NO ligand and Phe29 causing the side chain to shift towards His64, which, in turn, is pushed away from the NO-binding site (Figure 6, lower row). This reorientation of His64 breaks the helix and changes the hydrophobic side chain packing. Therefore the increase in stability observed in the ligand-bound form, might originate from favorable stacking interaction between the two aromatic rings (Phe29 and His64). Time resolved X-ray studies ${ }^{68}$ for the L29F mutant of MbCO found a concerted movement of Phe29 and His64 with concomitant displacement of His64 away from heme-bound CO towards the solvent which support the present findings (see also Figure 7).

It has been found that Mb sacrifices protein folding stability (reduced thermodynamic stability of WT compared with His64 mutants) for functionality (affinity to binding of $\mathrm{O}_{2}$ and discrimination against CO). Specifically, mutation of His64 to aliphatic or aromatic side chains invariably increases the thermodynamic stability of apo-Mb. ${ }^{61}$ This is consistent with the present findings for H64F $(-0.54 \mathrm{kcal} / \mathrm{mol})$ and $\mathrm{H} 64 \mathrm{~V}(-0.56 \mathrm{kcal} / \mathrm{mol})$ which compare 
quite favourably with experiment, see Table 2. For H64F the substitution of His64 (basic) by Phe64 (hydrophobic aromatic) leads to favorable hydrophobic packing between the helix E region and the molten globule core, which explains the stabilization of ligand-free Mb. ${ }^{61}$ For the H64V mutation substitution of His by a hydrophobic residue leads to favorable hydrophobic packing between the helix E-region which rationalizes the stabilization of the apo form. ${ }^{61}$

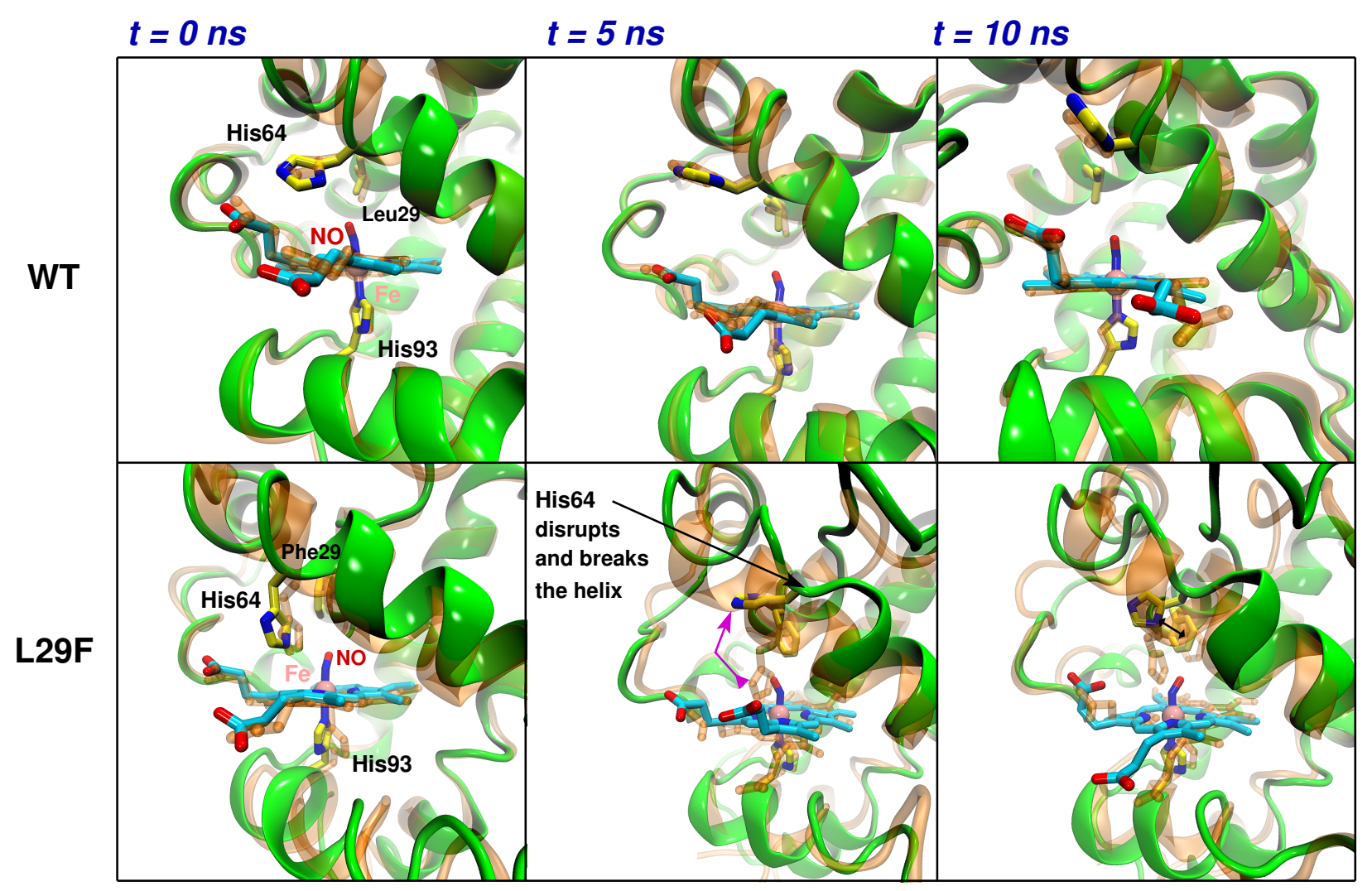

Figure 6: Conformational rearrangement of $\mathrm{Mb}$ with the heme group in both ligand-free (transparent orange) and ligand-bound (green) form as a function of the L29F mutation at different simulation time. Upper row: superposition of the ligand-free vs. the ligandbound (NO-bound) form in WT myoglobin at 0,5 and 10 ns of production MD. Lower row: superposition of the ligand-free vs. the ligand-bound form in L29F mutant at 0, 5 and 10 ns of production run. Representations: protein, new cartoon; His64, Leu/Phe29, Heme and $\mathrm{NO}$, licorice; Fe, vdw.

On the other hand, ligand-bound $\mathrm{H} 64 \mathrm{~F}$ and $\mathrm{H} 64 \mathrm{~V}$ are destabilized by 5.25 and $2.30 \mathrm{kcal} / \mathrm{mol}$, respectively. For ligand-bound H64F the presence of NO aggravates the situation since Phe64 is located at the entrance of the heme binding pocket and Phe at position 64 blocks the bind- 
ing site entry. Thus, for the H64F mutant the heme becomes restrained and the presence of NO causes a deformation of the Heme (see Figure 3 in SI). The differential stabilizations for $\mathrm{H} 64 \mathrm{~V}$ is $\delta \Delta \Delta G_{\text {stab }}=-2.9 \mathrm{kcal} / \mathrm{mol}$ compared with $\delta \Delta \Delta G_{\text {stab }}=-5.8 \mathrm{kcal} / \mathrm{mol}$ for the H64F mutant. As the results in Table 2 show, the computations are able to correctly describe stabilizing and destabilizing effects for ligand-free Mb and - within the error bars even yield quantitative agreement with experiment. ${ }^{61,65,66}$

Finally, the V68F mutation leads to ligand-free Mb which is more stable than WT by -1.05 kcal/mol. The replacement of the aliphatic Val by a bulky hydrophobic aromatic residue (Phe) leads to space restrictions around the heme and creates a more hydrophobic distal heme pocket that stabilizes the native state. ${ }^{61}$ Interestingly, the same stabilizing effect due to perpendicular $\pi$-stacking is found for the ligand-bound form and it is even more pronounced $(-3.40 \mathrm{kcal} / \mathrm{mol})$. This can be rationalized by the fact that residue 68 falls closer to the depth of the pocket and so the heme is not blocked at the entry and can adjust and adopt favorable structural rearrangements in the presence of NO (see Figure 4 in SI). These conclusions are also supported by previous studies where the contribution of the heme affinity to the stability of myoglobin was determined quantitatively. ${ }^{69}$ 


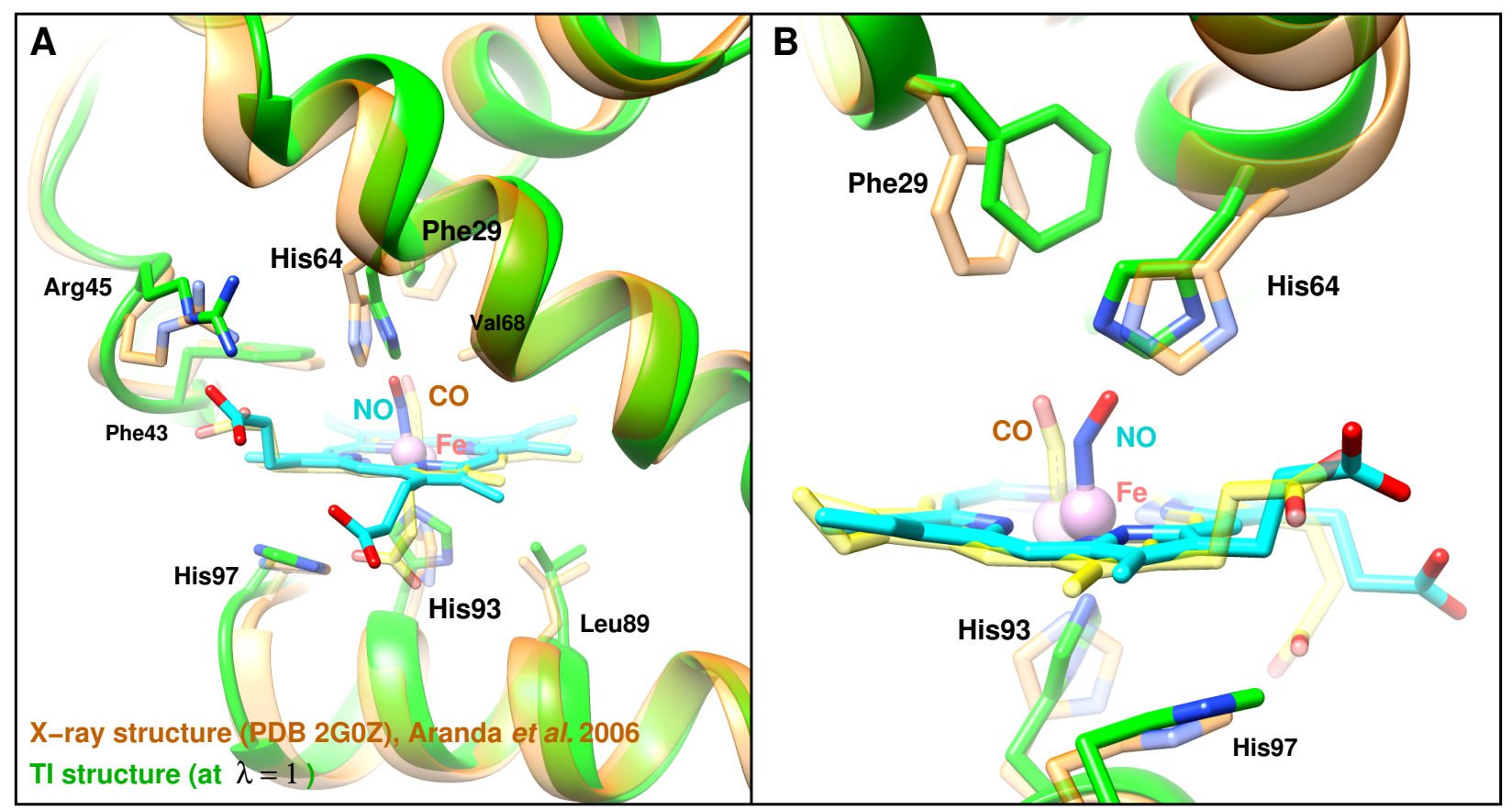

Figure 7: Superposition of NO-bound L29F structures from experiment (X-ray structure, PDB code: 2G0Z; ${ }^{68}$ CO-bound) (in transparent orange) and from the TI simulations (structure sampled at $\lambda=1$; NO-bound) (in green). (A) Shows the entire heme pocket with the important residues, and (B) emphasizes on residues Phe29 and His64. Representations: protein, new cartoon; important residues like His64, Leu/Phe29, Heme NO and CO, licorice; Fe, vdw.

Complementary to the TI simulations, thermodynamic stabilities were also determined by "iStable" 67 for all ligand-free Mb considered here. This software uses structural data and machine learning techniques to obtain a consensus score from several independent protein stability assessment schemes to predict the change in thermodynamic stability between the WT and a single point mutant. Using the 1U7S reference structure (holo-Mb), the predicted stability changes for the L29F, L29V, H64F, H64V, and V68F mutants are compatible with the results from TI (see Table 2), except for the V68F mutant.

\section{Discussion and Conclusion}

Prediction of $\Delta$ based on thermodynamics: The data for the H64V mutant suggests that 
values of $\Delta$ which generate the correct ligand rebinding kinetics $(\Delta=-10.25 \mathrm{kcal} / \mathrm{mol}$ compared to $\Delta=-7.25 \mathrm{kcal} / \mathrm{mol}$ for the $\mathrm{WT}$, i.e. a decrease of $-3.0 \mathrm{kcal} / \mathrm{mol}$ ) also lead to the correct differential stabilization of ligand-free and ligand-bound $\mathrm{Mb}$ of $-2.9 \mathrm{kcal} / \mathrm{mol}$. In other words, an appropriate value for the asymptotic separation $\Delta$ of the energy manifolds of the ${ }^{2} A$ and ${ }^{4} A$ states links (rebinding) kinetics and thermodynamic stability. In order to further investigate and quantify this qualitative and potential relationship two additional mutants were considered, L29F and V68F. First, the value for $\delta \Delta$ was determined from the differential stabilization between the ligand-free and ligand-bound state obtained by thermodynamic integration $\left(\delta \Delta=\delta \Delta \Delta G_{\text {statesstab }}\right)$ and in a next step these $\delta \Delta$ values were used to infer the change in asymptotic separation $\Delta$ relative to WT $\left(\Delta=\delta \Delta+\Delta_{\mathrm{WT}}\right)$ to determine the rebinding kinetics from 750 separate, individual rebinding simulations.

Table 3: Rebinding times, amplitudes and rebinding fraction within 200 ps for the L29F and V68F mutants for different values of $\Delta$.

\begin{tabular}{|l|r|r|r|r|r|r|}
\hline $\mathrm{Mb}$ & $\Delta$ & $a_{1}$ & $\tau_{1}(\mathrm{ps})$ & $a_{2}$ & $\tau_{2}(\mathrm{ps})$ & $\% N(t)(200 \mathrm{ps})$ \\
\hline $\mathrm{V} 68 \mathrm{~F}$ & -10.25 & 0.42 & 14.0 & 0.58 & 85.0 & $93.2 \pm 1.1$ \\
V68F & -5.25 & 0.71 & 7.8 & 0.29 & 94.2 & $94.8 \pm 1.0$ \\
Experiment & & 0.78 & $8.0-10.1$ & 0.21 & $35.0-60.2$ & 98.3 \\
\hline $\mathrm{L} 29 \mathrm{~F}$ & -10.25 & 0.48 & 8.4 & 0.52 & 155.5 & $85.4 \pm 1.5$ \\
$\mathrm{~L} 29 \mathrm{~F}$ & -8.25 & 0.56 & 6.4 & 0.44 & 145.5 & $86.4 \pm 1.5$ \\
$\mathrm{~L} 29 \mathrm{~F}$ & -6.25 & 0.60 & 6.6 & 0.40 & 132.1 & $90.0 \pm 1.3$ \\
L29F & -5.25 & 0.61 & 5.8 & 0.39 & 142.0 & $88.4 \pm 1.4$ \\
Experiment & & 0.95 & $6.0-6.7$ & 0.03 & 200.0 & 96.9 \\
\hline
\end{tabular}

Thermodynamic integration for the V68F mutant suggests that the relative stability change of ligand-free- and ligand-bound $\mathrm{Mb}$ for the mutant relative to the $\mathrm{WT}$ is $\delta \Delta \Delta G_{\text {stab }}=-2.4$ $\mathrm{kcal} / \mathrm{mol}$ (see Table 2). Therefore, rebinding dynamics simulations were run for $\Delta=-5.25$ $\mathrm{kcal} / \mathrm{mol}$ which is $2 \mathrm{kcal} / \mathrm{mol}$ lower than the value $(\Delta=-7.25 \mathrm{kcal} / \mathrm{mol})$ for $\mathrm{WT}$ which yields good agreement with experiment (see Figure 5). The NO-rebinding dynamics for the V68F mutant for both values of $\Delta$ is summarized in Table 3. The rebinding fraction after 200 ps for both values of $\Delta$ are quite similar (93\% vs. $95 \%$ ) and compare favourably with 
experiment $(98 \%)$. For the short time scale $\tau_{1}$ the simulations with $\Delta=-5.25 \mathrm{kcal} / \mathrm{mol}$ are in better agreement with experiment (7.8 ps compared with 8.0 to 10.1 ps from experiment) than those for $\Delta=-10.25 \mathrm{kcal} / \mathrm{mol}(14 \mathrm{ps})$. The long rebinding time scales $\tau_{2}$ are similar to one another but somewhat longer than those measured (85 ps and 94 ps compared to a value between $35 \mathrm{ps}$ and $60 \mathrm{ps}$ ). What really differs between the simulations with the two values for $\Delta$ is the amplitude of the rapidly rebinding fraction which increases from $42 \%$ $(\Delta=-10.25 \mathrm{kcal} / \mathrm{mol})$ to $71 \%(\Delta=-5.25 \mathrm{kcal} / \mathrm{mol})$ compared with $78 \%$ from experiment. Overall, the simulations with the lower value for $\Delta$ better describe the rebinding dynamics after photodissociation as compared with experiment.

Similarly, for the $\mathrm{L} 29 \mathrm{~F}$ mutant the $\delta \Delta \Delta G$ value of $1.1 \mathrm{kcal} / \mathrm{mol}$ suggests that rebinding dynamics simulations with $\Delta=-6.25 \mathrm{kcal} / \mathrm{mol}$ should yield improved ligand rebinding kinetics. As is shown in Table 3 the reference value of $\Delta=-10.25 \mathrm{kcal} / \mathrm{mol}$ leads to reasonable agreement for the two rebinding time scales and amplitudes and the rebinding fraction. However, decreasing $\Delta$ to between $-8.25 \mathrm{kcal} / \mathrm{mol}$ and $-5.25 \mathrm{kcal} / \mathrm{mol}$ improves the rebinding fraction, the short time scale $\tau_{1}$ and the amplitudes of both rebinding processes. Only for $\tau_{2}$ a somewhat less satisfactory result is found which, however, is the minority component (3\%) in the experiment. Nevertheless, the improvement by decreasing $\Delta$ according to the change in thermodynamic stabilisation is not quite as good as for the V68F mutant.

Structural Interpretation: Previously it was found that the long and short time scales for the ligand rebinding process are due to discrete conformational (sub-)states sampled by the system. ${ }^{22}$ Furthermore, different rebinding time scales are often associated with different free energy barriers $\Delta G^{\ddagger}$ between the initial (NO dissociated) and final states (NO bound). This implies that either $\Delta H, \Delta S$, or both change depending on the value of $\Delta$. In order to address this point the configurational space $(R, \theta)$ sampled by the dissociated ligand before rebinding is considered a) for the WT, V68F, H64V, and L29F mutants, respectively (Figure 8) 

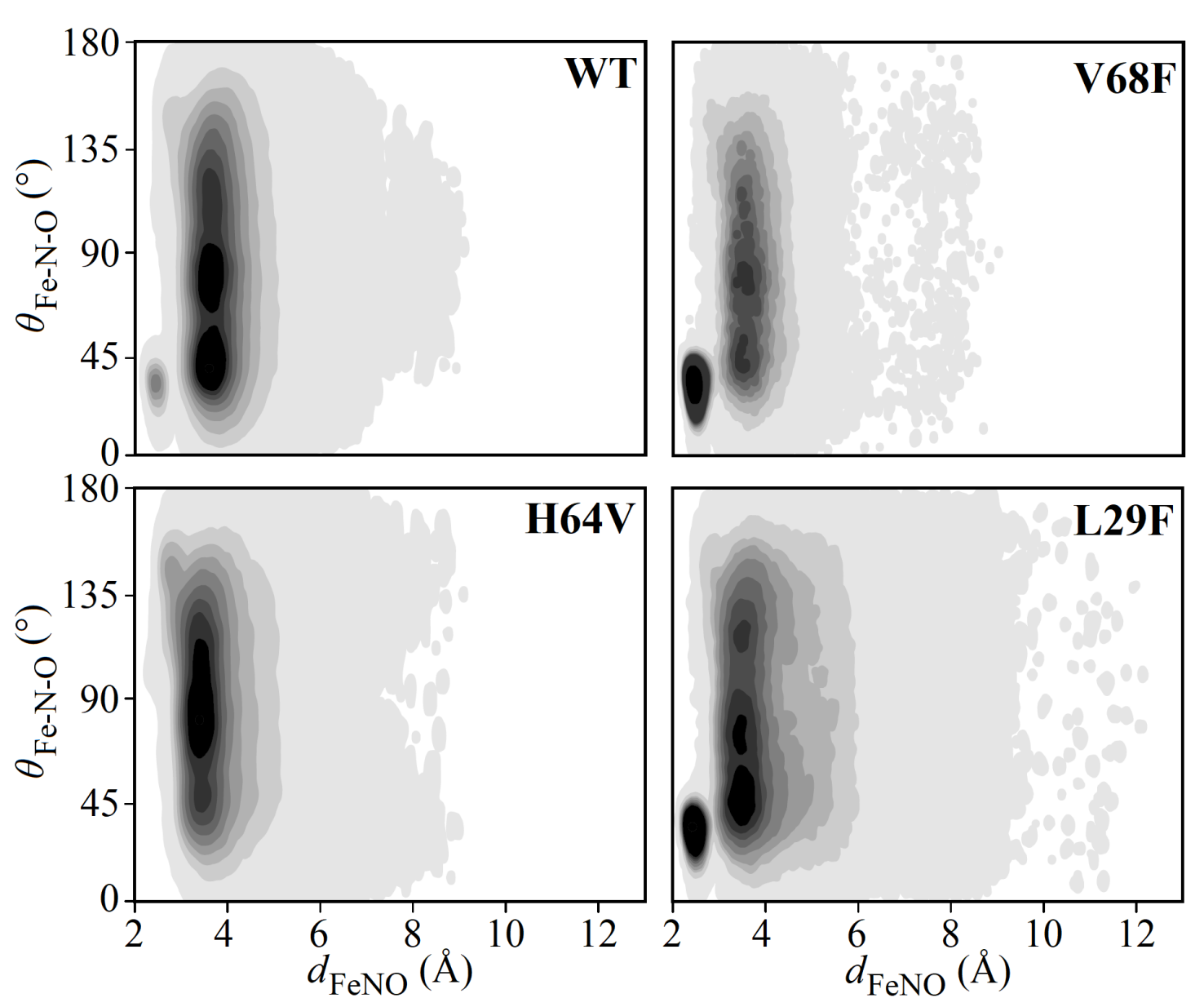

and b) for the same mutant (V68F) but for two values of the asymptotic shift $\Delta$, see Figure 9.

Figure 8: Comparison of ( $R$ (Fe-NO distance), $\theta$ (Fe-N-O angle) ) distributions for wild-type vs. mutant $\mathrm{Mb}$ after photodissociation. All simulations are carried out with the $\Delta$-value which leads to rebinding kinetics consistent with experiment. WT (top left, $\Delta=-7.25$ $\mathrm{kcal} / \mathrm{mol}, f(\mathrm{FeON})=1.6 \%$ ), V68F (top right, $\Delta=-5.25 \mathrm{kcal} / \mathrm{mol}, f(\mathrm{FeON})=23.2$ \%), H64V (bottom left, $\Delta=-10.25 \mathrm{kcal} / \mathrm{mol}, f(\mathrm{FeON})=0.2 \%$ ), L29F (bottom right, $\Delta=-5.25 \mathrm{kcal} / \mathrm{mol}, f(\mathrm{FeON})=7.9 \%)$. Iso-contours in steps of $10 \%$ of the maximum density are shown. Darker regions correspond to high probabilities whereas lighter regions to lower probabilities. $f$ corresponds to the fraction of population that is in the Fe-ON geometry.

Depending on the residues lining the active site, the available space for the ligand and the topology of the probability distribution function change. This is reflected in the maxima of $P(R, \theta)$ which shift and in the number of maxima which can differ from one variant to the 
next. The short rebinding time scales for L29F, V68F, H64V, and WT are $\tau_{1}=5.8$ ps, 7.8 ps, $9.2 \mathrm{ps}$, and $12.9 \mathrm{ps}$ and the amplitude of the rapid rebinding process is largest for L29F and V68F which are mutations from "small" to more "bulky" side chains. It is also for these two mutants that the fraction of population of the metastable Fe-ON state is largest (23\% for $\mathrm{V} 68 \mathrm{~F}$ and $8 \%$ for $\mathrm{L} 29 \mathrm{~F}$ ). Hence, it is conceivable that the possibility to populate the metastable state contributes to more rapid rebinding and to increasing the probability for this process.

Turning to $P(x, y, z)$ for $\mathrm{V} 68 \mathrm{~F}$ and two values for $\Delta(-10.25 \mathrm{kcal} / \mathrm{mol}$ as the standard value and $-5.25 \mathrm{kcal} / \mathrm{mol}$ which reproduces the experimentally observed kinetics, see Table 3 ) it is found that the projection $P(x, z)$ parallel to the heme plane for the two values of $\Delta$ is rather similar (see Figure 9). On the other hand, the projections $P(x, y)$ and $P(y, z)$ have additional favourable regions that can only be populated for $\Delta=-5.25 \mathrm{kcal} / \mathrm{mol}$. Because these concern regions closer to the iron atom (which is located in $(0,0,0)$ ) they influence the rebinding kinetics and contribute to the rapid phase as is found in the reactive MD simulations. The time scale $\tau_{1}$ shortens from $14.0 \mathrm{ps}$ to $7.8 \mathrm{ps}$ and its amplitude increases from $42 \%$ to $71 \%$. It is found that $\Delta$ affects the available space for the ligand - see Figure 9. For the same ligand (here NO) the enthalpic part is largely unchanged; hence what is affected in the change of the rate, i.e. the free energy barrier, is the entropic part.

In the present work NO-recombination primarily from positions within the active site, including docking site B, ${ }^{11,70}$ was investigated. Nevertheless, as the rebinding fractions ranging from $30 \%$ to $98 \%$ within the first 200 ps indicate (see Tables 1 and 3), the photodissociated ligand samples other regions of the protein interior. They include sites such as the Xe-pockets ${ }^{71-73}$ from where the ligand rebinds on longer scales, though, and were not further analyzed in the present work. 

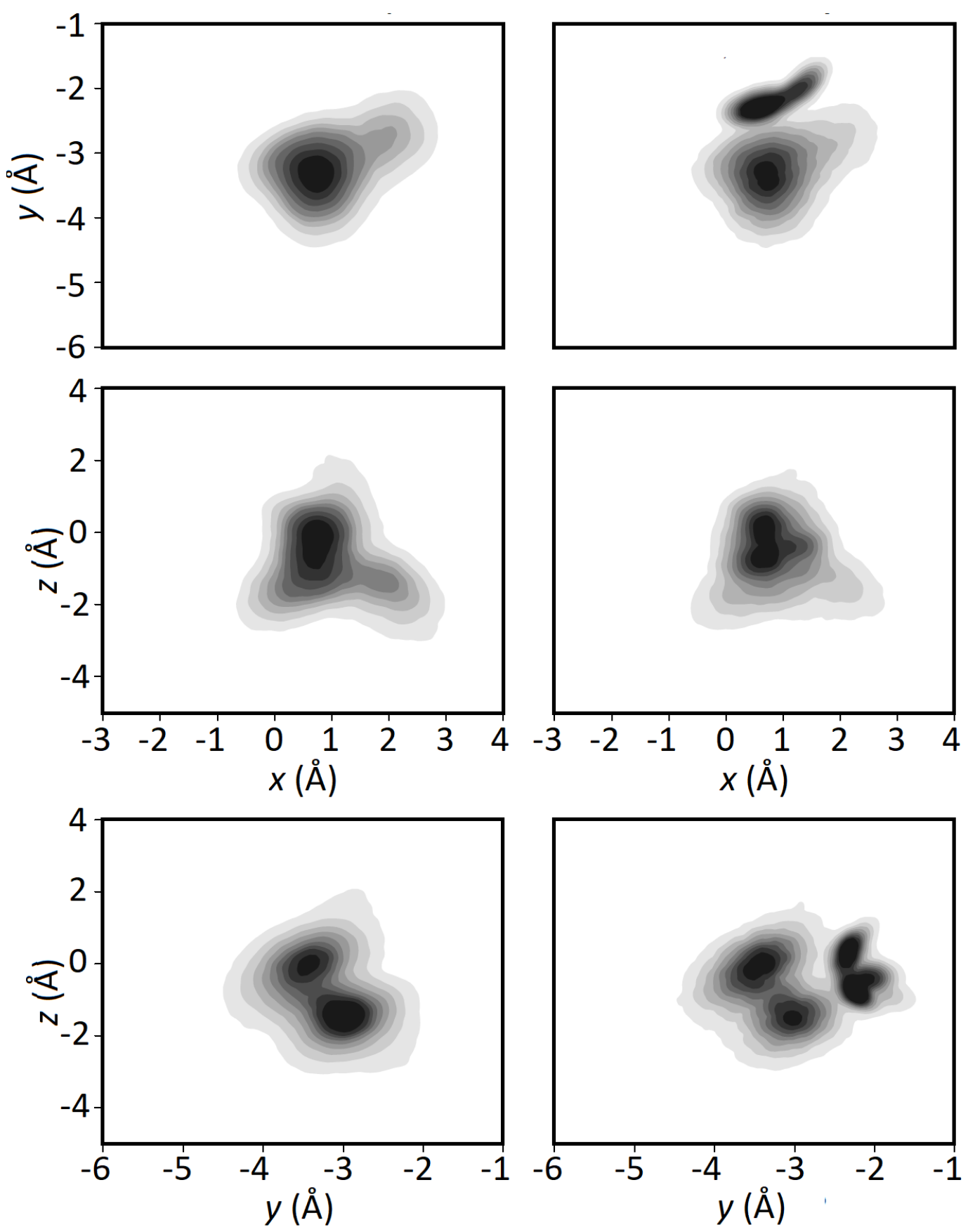

Figure 9: Influence of the asymptotic shift $\Delta$ on the conformational ensemble sampled by the dissociated ligand for the V68F mutant. The average heme-plane is in the $x y$ - plane with the iron atom in $(0,0,0)$. From top to bottom cuts through the $x y-, x z-$, and $y z$-planes. Left column for $\Delta=-10.25$ and right column for $\Delta=-5.25 \mathrm{kcal} / \mathrm{mol}$. For the lower $\Delta$-value the $\mathrm{Fe}-\mathrm{ON}$ state, which has the NO ligand considerably closer to the heme-iron $(y \sim-2 \AA)$, becomes accessible (see Figure 2$)$ which leads to the rapid rebinding time scale $\left(\tau_{1}=7.8 \mathrm{ps}\right)$. 
Previous synthetic and computational work suggested that the Fe-ON linkage isomer could be formed after photodissociation in MbNO. ${ }^{18,74}$ However, subsequent spectroscopic studies of WT, and five mutants (I28W, L108W, V68Y, H64L, and H64V) were unable to support this proposition. ${ }^{13}$ It is interesting to note that for WT the fraction of Fe-ON consistent with the rebinding kinetics is $\sim 2 \%$ whereas the state is not populated at all for $\mathrm{H} 64 \mathrm{~V}$ which agrees with these experiments. Hence, the concentration of Fe-ON may simply be too small to be detected in these experiments and for the systems studied. On the contrary, for mutants $\mathrm{L} 29 \mathrm{~F}$ and $\mathrm{V} 68 \mathrm{~F}$ the fraction of $\mathrm{Fe}-\mathrm{ON}$ consistent with the rebinding kinetics is considerably larger ( $8 \%$ and $23 \%$, respectively) and it would be interesting to consider these mutants when searching for the Fe-ON state.

Comparing WT and mutant MB, the NO-rebinding kinetics and the thermodynamics of ligand-free vs. ligand-bound Mb suggests that rebinding kinetics and thermodynamic stability are related. The structural interpretation of the rebinding time scales indicates that changes in the asymptotic separation $\Delta$ affect the internal conformational space available to the ligand as they directly modulate the volumes of the internal cavities. The general finding that internal pocket volume is inversely related to thermodynamic stability (smaller volume leads to increased stability) is consistent with NMR-work on WT and L99A mutant Lysozyme. ${ }^{75}$ Here, it is found that V68F is thermodynamically more stable than L29F for both, ligand-free and ligand-bound $\mathrm{Mb}$ and for the ligand-free-forms the computations are in good agreement with experiment. This apparently leads to a more compact binding site which is reflected in a reduction of the available configurational space for the dissociated ligand to sample, see Figure 8. This, in turn may be reflected in the larger rebinding fraction of 94.8 \% for V68F vs. 88.4 \% for L29F (from experiment: $98.3 \%$ vs. $96.9 \%$ ) within 200 ps.

The relationship between protein stability and kinetics for WT and mutants has been in- 
vestigated in detail for protein folding. ${ }^{30}$ In this context it is interesting to remark that the value of $\Phi_{\mathrm{F}}$ for folding has been found to be mutation-specific because "..each mutation removes different interactions and has different effects on the denatured state[..]". ${ }^{31}$ In the present work this is reflected by the fact that for each mutant a slightly different value for the asymptotic separation $\Delta$ is required (starting from a reference value for the WT) in order to correctly describe the ligand rebinding kinetics in the Mb mutants. The required changes in $\Delta$ for the mutants relative to WT as the reference are $\sim 2 \mathrm{kcal} / \mathrm{mol}$ which is consistent with typical thermodynamic stability changes upon mutation for Mb which span a range from -2 $\mathrm{kcal} / \mathrm{mol}$ to $+4 \mathrm{kcal} / \mathrm{mol}^{26}$

In summary, it is established that the rebinding kinetics of NO to Mb can be quantitatively characterized from atomistic simulations by adjusting one single, conformationally averaged quantity: the asymptotic energy separation $\Delta$ of the energy manifolds. Because each mutation removes (or introduces) different local and potentially nonlocal interactions it is evident that the "best" value of $\Delta$ for a particular mutant (relative to the "canonical value" of $\Delta$ for the WT protein) slightly differs depending on the protein variant considered. Most importantly, the same computational model can be used to determine the differential stability of the ligand-free- and ligand-bound form of the protein. Thus, for Mb a direct link between kinetics and thermodynamics for ligand rebinding dynamics and differential protein stability is found, akin to the relationship from $\Phi$-value analysis between protein stability and protein folding kinetics.

\section{Acknowledgments}

This work was supported by the Swiss National Science Foundation grant 200021-117810, the NCCR MUST, and the University of Basel. The authors thank Prof. J. Olson for fruitful 
discussions.

\section{Supporting Information}

The supporting information contains four figures illustrating the trajectory of transformation corresponding to the mutation (via the thermodynamic integration protocol) of wild-type myoglobin to the L29V (Figure 1), L29F (Figure 2), H64F (Figure 3) and V68F (Figure 4) mutants. This document also includes a comparison of 2-dimensional $(R, \theta)$ distributions for WT vs V68F and L29F myoglobins for the default and the experimentally relevant $\Delta$ values (Figure 5). This material is available free of charge via the Internet at http://pubs.acs.org. 


\section{References}

(1) Springer, B. A.; Sligar, S. G.; Olson, J. S.; Phillips, G. N. Mechanisms of Ligand Recognition in Myoglobin. Chem. Rev. 1994, 94, 699-714.

(2) Olson, J. S.; Phillips, G. N. Kinetic pathways and barriers for ligand binding to myoglobin. J. Biol. Chem. 1996, 271, 17593-17596.

(3) Danielsson, J.; Banushkina, P.; Nutt, D. R.; Meuwly, M. Computer simulations of structures, energetics and dynamics of myoglobin ligand complexes. Int. Rev. Phys. Chem. 2006, 25, 407-425.

(4) Olson, J. S.; Soman, J.; Phillips, G. N., Jr. Ligand pathways in myoglobin: A review of Trp cavity mutations. IUBMB Life 2007, 59, 552-562.

(5) Nienhaus, K.; Nienhaus, G. U. Comparison of ligand migration and binding in heme proteins of the globin family. Chin. Phys. B 2015, 24, article no: 128705.

(6) Petrich, J. W.; Lambry, J. C.; Kuczera, K.; Karplus, M.; Poyart, C.; Martin, J. L. Ligand-Binding and Protein Relaxation in Heme-Proteins - A Room-Temperature Analysis of NO Geminate Recombination. Biochemistry 1991, 30, 3975-3987.

(7) Gibson, Q. H.; Regan, R.; Elber, R.; Olson, J. S.; Carver, T. E. Distal Pocket Residues Affect Picosecond Ligand Recombination in Myoglobin - An Experimental and Molecular-Dynamics Study of Position 29 Mutants. J. Biol. Chem. 1992, 26r\%, $22022-22034$.

(8) Petrich, J. W.; Lambry, J. C.; Balasubramanian, S.; Lambright, D. G.; Boxer, S. G.; Martin, J. L. Ultrafast Measurements of Geminate Recombination of NO with SiteSpecific Mutants of Human Myoglobin. J. Mol. Biol. 1994, 238, 437-444.

(9) Shreve, A. P.; Franzen, S.; Simpson, M. C.; Dyer, R. B. Dependence of NO recombi- 
nation dynamics in horse myoglobin on solution glycerol content. J. Phys. Chem. B. 1999, 103, 7969-7975.

(10) Ye, X.; Demidov, A.; Champion, P. M. Measurements of the photodissociation quantum yields of $\mathrm{MbNO}$ and $\mathrm{MbO}(2)$ and the vibrational relaxation of the six-coordinate heme species. J. Am. Chem. Soc. 2002, 124, 5914-5924.

(11) Nienhaus, K.; Deng, P. C.; Olson, J. S.; Warren, J. J.; Nienhaus, G. U. Structural dynamics of myoglobin - Ligand migration and binding in valine 68 mutants. J. Biol. Chem. 2003, 278, 42532-42544.

(12) Kim, S.; Jin, G.; Lim, M. Dynamics of geminate recombination of NO with myoglobin in aqueous solution probed by femtosecond mid-IR spectroscopy. J. Phys. Chem. B. 2004, 108, 20366-20375.

(13) Nienhaus, K.; Palladino, P.; Nienhaus, G. U. Structural dynamics of Myoglobin: FTIRTDS study of NO migration and binding. Biochem. 2008, 47, 935-948.

(14) Kruglik, S. G.; Yoo, B.-K.; Franzen, S.; Vos, M. H.; Martin, J.-L.; Negrerie, M. Picosecond primary structural transition of the heme is retarded after nitric oxide binding to heme proteins. Proc. Natl. Acad. Sci. U.S.A. 2010, 107, 13678-13683.

(15) Kim, S.; Lim, M. Protein Conformation-Controlled Rebinding Barrier of NO and Its Binding Trajectories in Myoglobin and Hemoglobin at Room Temperature. J. Phys. Chem. B 2012, 116, 5819-5830.

(16) Silatani, M.; Lima, F. A.; Penfold, T. J.; Rittmann, J.; Reinhard, M. E.; RittmannFrank, H. M.; Borca, C.; Grolimund, D.; Milne, C. J.; Chergui, M. NO binding kinetics in myoglobin investigated by picosecond Fe K-edge absorption spectroscopy. Proc. Natl. Acad. Sci. U.S.A. 2015, 112, 12922-12927. 
(17) Li, H. Y.; Elber, R.; Straub, J. E. Molecular-Dynamics Simulation of NO Recombination to Myoglobin Mutants. J. Biol. Chem. 1993, 268, 17908-17916.

(18) Nutt, D. R.; Karplus, M.; Meuwly, M. Potential energy surface and molecular dynamics of MbNO: Existence of an unsuspected FeON minimum. J. Phys. Chem. B 2005, 109, $21118-21125$.

(19) Nutt, D. R.; Meuwly, M. Studying reactive processes with classical dynamics: Rebinding dynamics in MbNO. Biophys. J. 2006, 90, 1191-1201.

(20) Cottone, G.; Lattanzi, G.; Ciccotti, G.; Elber, R. Multiphoton Absorption of Myoglobin-Nitric Oxide Complex: Relaxation by D-NEMD of a Stationary State. J. Phys. Chem. B 2012, 116, 3397-3410.

(21) Soloviov, M.; Meuwly, M. Reproducing kernel potential energy surfaces in biomolecular simulations: Nitric oxide binding to myoglobin. J. Chem. Phys. 2015, 143, 105103.

(22) Soloviov, M.; Das, A. K.; Meuwly, M. Structural Interpretation of Metastable States in Myoglobin-NO. Angew. Chem. Int.. Ed. 2016, 55, 10126-10130.

(23) Wang, Y. H.; Baskin, J. S.; Xia, T. B.; Zewail, A. H. Human myoglobin recognition of oxygen: Dynamics of the energy landscape. Proc. Natl. Acad. Sci. U.S.A. 2004, 101, $18000-18005$.

(24) Kholodenko, Y.; Gooding, E. A.; Dou, Y.; Ikeda-Saito, M.; Hochstrasser, R. M. Heme protein dynamics revealed by geminate nitric oxide recombination in mutants of iron and cobalt myoglobin. Biochemistry 1999, 38, 5918-5924.

(25) Shoichet, B. K.; Baase, W. A.; Kuroki, R.; W., M. B. A Relationship Between Protein Stability and Protein Function. Proc. Nat. Acad. Sci. U.S.A. 1995, 92, 452-456.

(26) Kepp, K. P. Towards a Golden Standard for computing globin stability: Stability and 
structure sensitivity of myoglobin mutants. Biochim. Biophys. Acta-Proteins and Proteomics 2015, 1854, 1239-1248.

(27) Leffler, J. E. Papameters for the Description of Transition States. Science 1953, 117, $340-341$.

(28) Bronsted, J. N.; Pedersen, K. The catalytic disintegration of nitramide and its physicalchemical relevance. Z. Physik. Chem. 1924, 108, 185-235.

(29) Hammett, L. P. The effect of structure upon the reactions of organic compounds benzene derivatives. J. Am. Chem. Soc. 1937, 59, 96-103.

(30) Matouschek, A.; Kellis, J. T.; Serrano, L.; Fersht, A. R. Mapping the Transition State and Pathway of Protein Folding by Protein Engineering. Nature 1989, 340, 122-126.

(31) Fersht, A. R.; Sato, S. Phi-Value analysis and the nature of protein-folding transition states. Proc. Natl. Acad. Sci. 2004, 101, 7976-7981.

(32) Brooks, B. R.; Bruccoleri, R. E.; Olafson, B. D.; States, D. J.; Swaminathan, S.; Karplus, M. CHARMM - A Program for Macromolecular Energy, Minimization, and Dynamics Calculations. J. Comput. Chem. 1983, 4, 187-217.

(33) Brooks, B. R.; Brooks, C. L., III; Mackerell, A. D., Jr.; Nilsson, L.; Petrella, R. J.; Roux, B.; Won, Y.; Archontis, G.; Bartels, C.; Boresch, S. et al. CHARMM: The Biomolecular Simulation Program. J. Comput. Chem. 2009, 30, 1545-1614.

(34) MacKerell, A. D.; Bashford, D.; Bellott, M.; Dunbrack, R. L.; Evanseck, J. D.; Field, M. J.; Fischer, S.; Gao, J.; Guo, H.; Ha, S. et al. All-atom empirical potential for molecular modeling and dynamics studies of proteins. J. Phys. Chem. B 1998, 102, 3586-3616.

(35) Mackerell Jr., A. D.; Feig, M.; Brooks, C. L. Extending the treatment of backbone energetics in protein force fields: Limitations of gas-phase quantum mechanics in re- 
producing protein conformational distributions in molecular dynamics simulations. J. Comput. Chem. 2004, 25, 1400-1415.

(36) Jorgensen, W. L.; Chandrasekhar, J.; Madura, J. D.; Impey, R. W.; Klein, M. L. Comparison of Simple Potential Functions for Simulating Liquid Water. J. Chem. Phys. 1983, 79, 926-935.

(37) VanGunsteren, W. F.; Berendsen, H. J. C. Algorithms for Macromolecular Dynamics and Constraint Dynamics. Mol. Phys. 1977, 34, 1311-1327.

(38) Ryckaert, J. P.; Ciccotti, G.; Berendsen, H. J. C. Numerical-Integration of Cartesian Equations of Motion of a System With Constraints - Molecular-Dynamics of N-Alkanes. J. Comput. Phys. 1977, 23, 327-341.

(39) Aronszajn, N. Theory of Reproducing Kernels. Trans. Am. Math. Soc. 1950, 68, 337404.

(40) Hollebeek, T.; Ho, T. S.; Rabitz, H. Constructing multidimensional molecular potential energy surfaces from ab initio data. Annu. Rev. Phys. Chem. 1999, 50, 537-570.

(41) Banushkina, P.; Meuwly, M. Diffusive dynamics on multidimensional rough free energy surfaces. J. Chem. Phys. 2007, 127.

(42) Lim, M.; Jackson, T. A.; Anfinrud, P. A. Nonexponential protein relaxation: dynamics of conformational change in myoglobin. Proc. Natl. Acad. Sci. 1993, 90, 5801-5804.

(43) Zoete, V.; Meuwly, M. Importance of individual side chains for the stability of a protein fold: Computational alanine scanning of the insulin monomer. J. Comput. Chem. 2006, $27,1843-1857$.

(44) Boresch, S.; Karplus, M. The role of bonded terms in free energy simulations: 1. Theoretical analysis. J. Phys. Chem. A 1999, 103, 103-118. 
(45) Pearlman, D. A. A Comparison of Alternative Approaches to Free-Energy Calculations. J. Phys. Chem. 1994, 98, 1487-1493.

(46) Boresch, S. The role of bonded energy terms in free energy simulations-Insights from analytical results. Mol. Sim. 2002, 28, 13-37.

(47) Zacharias, M.; Straatsma, T. P.; McCammon, J. A. Separation-shifted scaling, a new scaling method for Lennard-Jones interactions in thermodynamic integration. J. Chem. Phys. 1994, 100, 9025-9031.

(48) Evans, D. J.; Holian, B. L. The NoseHoover thermostat. J. Chem. Phys. 1985, 83, 4069-4074.

(49) Hédin, F.; El Hage, K.; Meuwly, M. A Toolkit to Fit Nonbonded Parameters from and for Condensed Phase Simulations. J. Chem. Inf. Model. 2016, 56, 1479-1489.

(50) Bash, P. A.; Singh, U. C.; Brown, F. K.; Langridge, R.; Kollman, P. A. Calculation of the relative change in binding free energy of a protein-inhibitor complex. 1987, 235, 574-576.

(51) Schaad, O.; Zhou, H. X.; Szabo, A.; Eaton, W. A.; Henry, E. R. Simulation of the Kinetics of Ligand-Binding to a Protein by Molecular-Dynamics - Geminate Rebinding of Nitric-Oxide to Myoglobin. Proc. Natl. Acad. Sci. U.S.A. 1993, 90, 9547-9551.

(52) Ionascu, D.; Gruia, F.; Ye, X.; Yu, A. C.; Rosca, F.; Beck, C.; Demidov, A.; Olson, J. S.; Champion, P. M. Temperature-dependent studies of NO recombination to heme and heme proteins. J. Amer. Chem. Soc. 2005, 12\%, 16921-16934.

(53) Quillin, M. L.; Li, T. S.; Olson, J. S.; Phillips, G. N.; Dou, Y.; Ikedasaito, M.; Regan, R.; Carlson, M.; Gibson, Q. H.; Li, H. Y. et al. Structural and Functional-Effects of Apolar Mutations of the Distal Valine in Myoglobin. J. Mol. Biol. 1995, 245, 416-436. 
(54) Olson, J. S.; Phillips, G. N. Kinetic pathways and barriers for ligand binding to myoglobin. J. Biol. Chem. 1996, 271, 17593-17596.

(55) Baker, W. N. A new comparison of the viscosity of D2O with that of H2O. J. Chem. Phys. 1936, 4, 294-295.

(56) Mondal, P.; Meuwly, M. Solvent Composition Drives the Rebinding Kinetics of Nitric Oxide to Microperoxidase. Scientific Reports 2018, 8.

(57) Lecomte, J. T. J.; Lamar, G. N. H-1-NMR Probe for Hydrogen-Bonding of Distal Residues to Bound Ligands in Heme-Proteins - Isotope Effect on Heme ElectronicStructure of Myoglobin. J. Am. Chem. Soc. 1987, 109, 7219-7220.

(58) Mie, Y.; Yamada, C.; Uno, T.; Neya, S.; Mizutani, F.; Nishiyama, K.; Taniguchi, I. Notable deuterium effect on the electron transfer rate of myoglobin. Chem. Comm. 2005, 250-252.

(59) Unno, M.; Christian, J. F.; Olson, J. S.; Sage, J. T.; Champion, P. M. Evidence for hydrogen bonding effects in the iron ligand vibrations of carbonmonoxy myoglobin. $J$. Am. Chem. Soc. 1998, 120, 2670-2671.

(60) Gibson, Q.; Antonini, E. Kinetic Studies on the Reation between Native Globin and Haem Derivatives. Biochem. J. 1960, 77, 328-341.

(61) Hargrove, M. S.; Krzywda, S.; Wilkinson, A. J.; Dou, Y.; Ikeda-Saito, M.; Olson, J. S. Stability of Myoglobin: A Model for the Folding of Heme Proteins. Biochemistry 1994, 33, $11767-11775$.

(62) Barrick, D.; Baldwin, R. L. The molten globule intermediate of apomyoglobin and the process of protein folding. Protein Science 1993, 2, 869-876.

(63) Barrick, D.; Baldwin, R. L. Three-state analysis of sperm whale apomyoglobin folding. Biochemistry 1993, 32, 3790-3796. 
(64) Pinker, R. J.; Lin, L.; Kallenbach, N. R.; Rose, G. D. Effects of alanine substitutions in -helices of sperm whale myoglobin on protein stability. Protein Science 1993, 2, 1099-1105.

(65) Lin, L.; Pinker, R. J.; Kallenbach, N. R. .alpha.-Helix stability and the native state of myoglobin. Biochemistry 1993, 32, 12638-12643.

(66) Smith, L. The Effects of Amino Acid Substitution on Apomyoglobin Stability, Folding Intermediates, and Holoprotein Expression (PhD Thesis); Rice University: Houston, Texas, 2003.

(67) Chen, C.-W.; Lin, J.; Chu, Y.-W. iStable: off-the-shelf predictor integration for predicting protein stability changes. BMC Bioinformatics 2013, 14, S5.

(68) Aranda, R., IV; Levin, E. J.; Schotte, F.; Anfinrud, P. A.; Phillips, G. N., Jr Timedependent atomic coordinates for the dissociation of carbon monoxide from myoglobin. Acta Cryst. 2006, 62, 776-783.

(69) Hargrove, M. S.; Olson, J. S. The Stability of Holomyoglobin Is Determined by Heme Affinity. Biochemistry 1996, 35, 11310-11318.

(70) Nutt, D.; Meuwly, M. Ligand dynamics in myoglobin: Calculation of infrared spectra for photodissociated NO. Chem. Phys. Chem. 2004, 5, 1710-1718.

(71) Tilton, R. F.; Kuntz, I. D.; Petsko, G. A. Cavities in Proteins - Structure of a Metmyoglobin-Xenon Complex Solved to 1.9-A. Biochemistry 1984, 23, 2849-2857.

(72) Ostermann, A.; Waschipky, R.; Parak, F. G.; Nienhaus, G. U. Ligand binding and conformational motions in myoglobin. Nature 2000, 404, 205-208.

(73) Nutt, D. R.; Meuwly, M. Migration in native and mutant myoglobin: Atomistic simulations for the understanding of protein function. Proc. Natl. Acad. Sci. 2004, 101, 5998-6002. 
(74) Cheng, L.; Novozhilova, I.; Kim, C.; Kovalevsky, A.; Bagley, K. A.; Coppens, P.; Richter-Addo, G. B. First observation of photoinduced nitrosyl linkage isomers of iron nitrosyl porphyrins. J. Am. Chem. Soc. 2000, 122, 7142-7143.

(75) Nucci, N. V.; Fuglestad, B.; Athanasoula, E. A.; Wand, A. J. Role of cavities and hydration in the pressure unfolding of T-4 lysozyme. Proc. Natl. Acad. Sci. 2014, 111, $13846-13851$. 


\section{Table of Contents (TOC) Image}

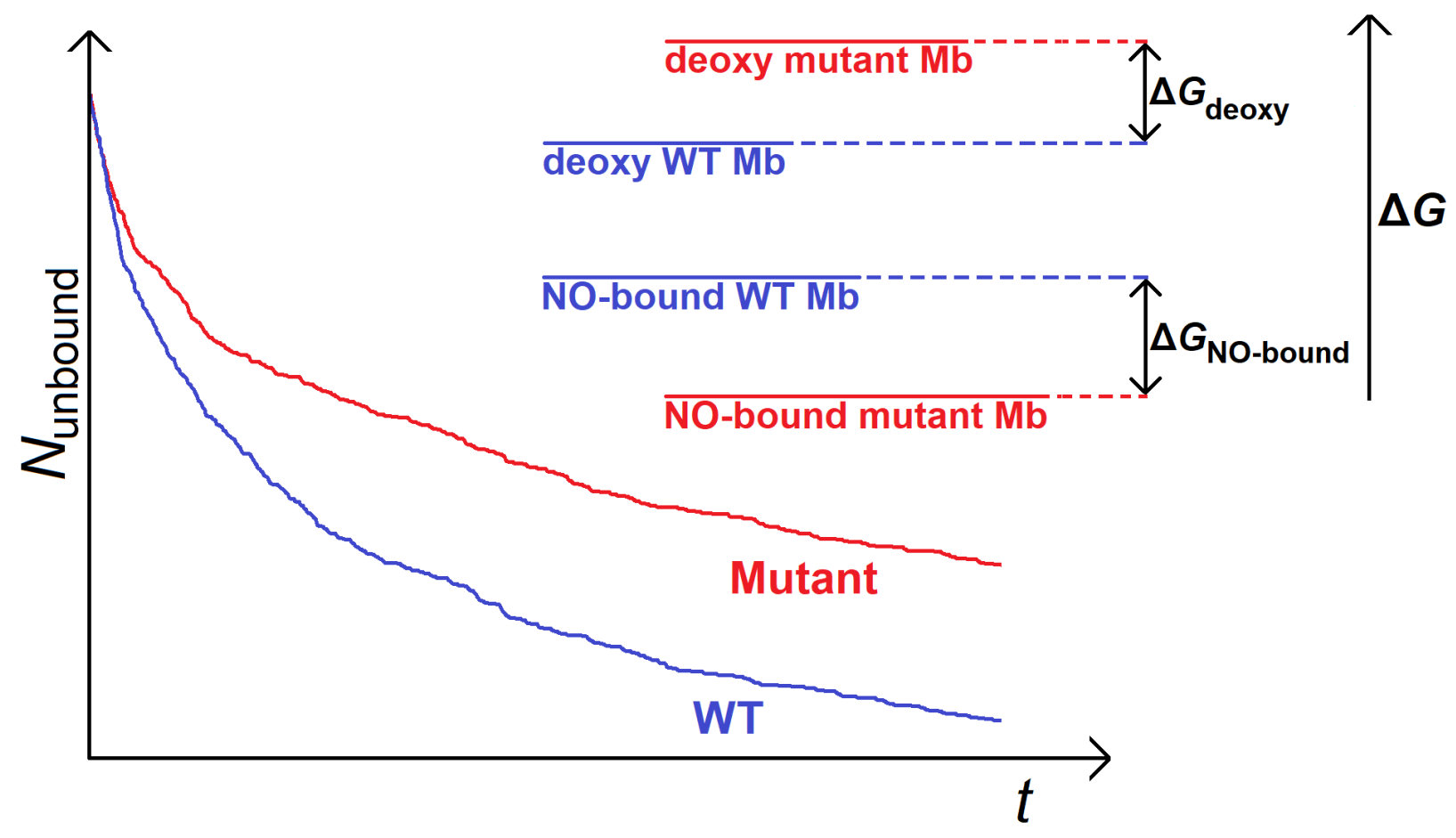

